OPEN ACCESS

Edited by:

Deyu Xie,

North Carolina State University,

United States

Reviewed by:

Md Abdur Rahim,

Sher-e-Bangla Agricultural University,

Bangladesh

James Robert Ketudat Cairns, Suranaree University of Technology,

Thailand

Masayoshi Nakayama National Agricultural Research

Organisation, Uganda

*Correspondence:

He Huang

101navy@163.com

Silan Dai

silandai@sina.com

${ }^{\dagger}$ These authors have contributed equally to this work

Specialty section:

This article was submitted to

Plant Metabolism

and Chemodiversity,

a section of the journal

Frontiers in Plant Science

Received: 12 December 2020 Accepted: 01 February 2021

Published: 22 February 2021

Citation

Lu C, Li Y, Cui Y, Ren J, Qi F, Qu J, Huang H and Dai S (2021) Isolation and Functional Analysis of Genes Involved in Polyacylated Anthocyanin Biosynthesis in Blue

Senecio cruentus.

Front. Plant Sci. 12:640746

doi: $10.3389 / f p / s .2021 .640746$

\section{Isolation and Functional Analysis of Genes Involved in Polyacylated Anthocyanin Biosynthesis in Blue Senecio cruentus}

\author{
Chenfei Lut, Yajun Lit, Yumeng Cui, Jiangshan Ren, Fangting Qi, Jiaping Qu, He Huang* \\ and Silan Dai*
}

Beijing Advanced Innovation Center for Tree Breeding by Molecular Design, Beijing Key Laboratory of Ornamental Plants Germplasm Innovation and Molecular Breeding, National Engineering Research Center for Floriculture, Beijing Laboratory of Urban and Rural Ecological Environment, Key Laboratory of Genetics and Breeding in Forest Trees and Ornamental Plants of Education Ministry, School of Landscape Architecture, Beijing Forestry University, Beijing, China

Polyacylated anthocyanins with multiple glycosyl and aromatic acyl groups tend to make flowers display bright and stable blue colours. However, there are few studies on the isolation and functional characterization of genes involved in the polyacylated anthocyanin biosynthesis mechanism, which limits the molecular breeding of truly blue flowers. Senecio cruentus is an important potted ornamental plant, and its blue flowers contain 3',7-polyacylated delphinidin-type anthocyanins that are not reported in any other plants, suggesting that it harbours abundant gene resources for the molecular breeding of blue flowers. In this study, using high-performance liquid chromatography-tandem mass spectrometry (HPLC-MS/MS) analysis of blue, carmine and white colours of cineraria cultivars "Venezia" (named VeB, VeC, and VeW, respectively), we found that 3',7-polyacylated anthocyanin, cinerarin, was the main pigment component that determined the blue colour of ray florets of cineraria. Based on the transcriptome sequencing and differential gene expression (DEG) analysis combined with RT- and qRT-PCR, we found two genes encoding uridine diphosphate glycosyltransferase, named SCUGT1 and ScUGT4; two genes encoding acyl-glucosidedependent glucosyltransferases which belong to glycoside hydrolase family $1(\mathrm{GH} 1)$, named ScAGGT11 and SCAGGT12; one gene encoding serine carboxypeptidase-like acyltransferase SCSCPL2; and two MYB transcriptional factor genes SCMYB2 and $S C M Y B 4$, that were specifically highly expressed in the ray florets of $\mathrm{VeB}$, which indicated that these genes may be involved in cinerarin biosynthesis. The function of ScSCPL2 was analysed by virus-induced gene silencing (VIGS) in cineraria leaves combined with HPLC-MS/MS. SCSCPL2 mainly participated in the $3^{\prime}$ and 7-position acylation of cinerarin. These results will provide new insight into the molecular basis of the polyacylated anthocyanin biosynthesis mechanism in higher plants and are of great significance for blue flower molecular breeding of ornamental plants.

Keywords: blue flower, polyacylated anthocyanins, Senecio cruentus, acyltransferase, glucosyltransferase 


\section{INTRODUCTION}

Flower colour is one of the most important traits of ornamental plants. Bright and stable blue flowers are precious and scarce resources in nature, and they are popular among consumers for their dream-like visual experience (Noda, 2018). Blue floral colour primarily arises from anthocyaninss, which are modified by aromatic or fatty acyl groups that are commonly linked to the hydroxy groups of the glycosyl moieties. Anthocyanins modified with multiple aromatic acyl groups are often referred to as polyacylated anthocyanins, and the colour shifts to blue gradually as the number of acyl groups increases (Yoshida et al., 2009; Tanaka and Brugliera, 2013). Meanwhile, polyacylation at the $3^{\prime}$ or 7-position of anthocyanin is more important for stable blue coloration, while the 3- or 5-position appears to provide only a reddish-purple colour (Matsuba et al., 2010; Nishizaki et al., 2013; Yoshida and Negishi, 2013). Although many different polyacylated anthocyanin structures have been identified from different species, isolation and functional studies of genes involved in polyacylation modification are few, which limits the molecular breeding of truly blue flowers.

The anthocyanin biosynthesis pathway in higher plants is one of the most clearly elucidated secondary metabolic pathways, mainly including the metabolic enzymes such as chalcone synthase (CHS), chalcone isomerase ( $\mathrm{CHI}$ ), flavanone 3-hydroxylase $(\mathrm{F} 3 \mathrm{H})$, flavonoid $3^{\prime}$-hydroxylase $\left(\mathrm{F}^{\prime} \mathrm{H}\right)$, flavonoid $3^{\prime}, 5^{\prime}$-hydroxylase $\left(\mathrm{F}^{\prime} 5^{\prime} \mathrm{H}\right)$, dihydroflavonol 4-reductase (DFR), anthocyanidin synthase (ANS), glycosyltransferase (GT), and acyltransferase (AT) (Liu et al., 2018; Amoanimaa-Dede et al., 2019). Polyacylation modification, which is crucial for blue flower coloration, is catalysed by GTs and ATs in an appropriate order, among which uridine diphosphate-dependent glycosyltransferase family 1 (UGT) and glycoside hydrolase family 1 (GH1) are two widely studied enzyme families known to transfer glycosyl groups (Sasaki and Nakayama, 2015). UGTs can transfer glycosyls from UDP sugars to flavonoids, and their catalytic function can be divided into 4 clades according to phylogenetic analysis. UGTs from Clades I and II catalyse the glycosylation of the 3- and 5-O-position of anthocyanins, respectively, which is the key step for the formation of stable anthocyanins and is also the most conventional and common glycosylation modification. UGTs from Clade III catalyse the glycosylation of $3^{\prime}$ or 7-O-position of flavonoids, which is involved in the biosynthesis of polyacylated anthocyanins (Nakajima et al., 2001; Yonekura-Sakakibara and Hanada, 2011; Sasaki and Nakayama, 2015). Acyl-glucoside-dependent glucosyltransferases (AGGTs), which belong to glycoside hydrolase family 1 (GH1), catalyse the glycosylation of anthocyanins with acyl-glucoses as glucosyl donors. AGGTs were identified for the first time in carnation and delphinium (named DcAA5GT and DgAA7GT, respectively), which are known to accumulate in vacuoles and contain strict substrate preferences (Luang et al., 2013; Matsuba et al., 2010).

Anthocyanin acyltransferase (AAT) is responsible for catalysing the acylation of anthocyanins after glycosyl connections. Serine carboxypeptidase-like (SCPL)-AATs are a type of enzymes that belong to the $\alpha / \beta$ hydrolase family. These enzymes have acyltransferase activity of secondary metabolites, involving advanced and even final modification of anthocyanins after being transported into the vacuole. Anthocyanins with multiple aromatic acyl groups (often referred to as polyacylated anthocyanins) are the main products catalysed by acyltransferase (Ciarkowska et al., 2018), and the stacking of aromatic acyl groups makes the anthocyanins appear blue. According to phylogenetic evolution, SCPLs can be clustered into three clades, Clade IA, Clade IB, and Clade II. Among them, members of Clade IA generally have acyltransferase activity, and the expression of these genes is specific to tissues and organs, while other SCPL genes are widely expressed in different plant tissues (Fraser et al., 2005). At present, SCPL genes involved in acylation of anthocyanins and other flavonoids have been identified from Clitoria ternatea, Delphinium grandiflorum, and Diospyros kaki (Noda et al., 2006; Milkowski and Strack, 2010; Akagi et al., 2011; Nishizaki et al., 2013). However, the mechanism for some glycosylation and acylation steps is variable in different species. Thus, clarifying more modification mechanisms and identifying more key genes will be conducive to the blue flower molecular breeding of ornamental plants.

In addition, the transcription of structural genes related to anthocyanin synthesis is mainly regulated by the MYBbHLH-WD40 (MBW) transcriptional complex, among which MYB transcription factors are the most important. Most studies of MYB found that it could regulate the entire pathway of anthocyanin metabolism. In recent years, many studies have found that different members of the MYB family are likely to carry out specific regulation on different branches of anthocyanin metabolism and biochemical modification (Nakatsuka et al., 2008; Chen et al., 2019). For example, in Hordeum vulgare and Gentiana triflora, MYB transcription factors can directly regulate the expression of F3' $5^{\prime} H$ (Nakatsuka et al., 2008; Jia et al., 2020). PpMYB9 and PpMYB10.2 in Prunus persica had transcriptional activation of the glycosylation genes PpUGT78A2 and PpUFGT, respectively (Hui et al., 2016). However, there are few studies of the transcriptional regulation mechanism of polyacylation modification in plants.

Cineraria (Senecio cruentus), which is an important potted flower in Compositae, is widely used in landscapes and has high ornamental and economic value. As the major pigment component in cineraria, Dp3MalG-7d(CafG)-3'CafG (cinerarin) has $3^{\prime}$ and 7-position polyacylation simultaneously (Suzuki et al., 2003; Sun et al., 2010; Sasaki and Nakayama, 2015). The $3^{\prime}$ and 7-position polyacylation of cinerarin is key for the bright blue coloration of cineraria. However, there are few reports of gene isolation, functional verification and metabolic regulation involved in this metabolic process, which limits the breeding of truly blue flowers. In this study, we analysed the pigment composition of blue, carmine and white cineraria cultivars "Venezia" by HPLC-MS/MS and screened out the key genes encoding glycolyltransferase and acyltransferase, as well as transcription factors that may be involved in the polyacylation modification of anthocyanins, using comparative transcriptomics and gene expression analysis. While the flower colour shifts to blue gradually as the number of acyl groups catalysed by acyltransferase increases (Yoshida et al., 2009; 
Tanaka and Brugliera, 2013), we further verified the function of the key structural gene ScSCPL2, which encodes a SCPL acyltransferase, by virus-induced gene silencing (VIGS) in cineraria leaves combined with HPLC-MS/MS. The results will provide new insight into the molecular basis of the polyacylated anthocyanin biosynthesis mechanism in higher plants and are of great significance for blue flower molecular breeding of ornamental plants.

\section{MATERIALS AND METHODS}

\section{Plant Material and Sampling}

The blue, white and carmine colours of cineraria cultivars 'Venezia' were chosen as plant materials, named VeB, VeW, and $\mathrm{VeC}$, respectively, in this study. Seedlings were purchased from Youshang Flower Co., Ltd. (Shanghai). As the ray florets developed, five different stages were determined according to previous study (Jin et al., 2016). At the S1 stage, the ray florets outgrew the bract with colour, the length of the protruding bract was less than the length of the whole bract, and the capitulums were contracted. At the S2 stage, the length of the protruding bract was approximately 1.5 times the length of the bract, and the capitulums tightly overlapped. At the S3 stage, the ray florets were obviously elongated and widened, with the disc florets exposed for the first time. At the S4 stage, the outgrowing angle of the ray florets was approximately $45^{\circ}$, and the disc florets were completely visible. At the S5 stage, the ray florets were fully opened, and the outermost disc florets began spreading pollen (Figure 1). In this study, mixed samples of disc florets, ray florets, leaves and stems of $\mathrm{VeB}$ were used for full-length transcriptome sequencing. In addition, the ray florets at the S1, S2, S4, and S5 stages of VeB and VeW were used fornext-generation transcriptome sequencing, and three biological replicates were taken at each developmental stage.

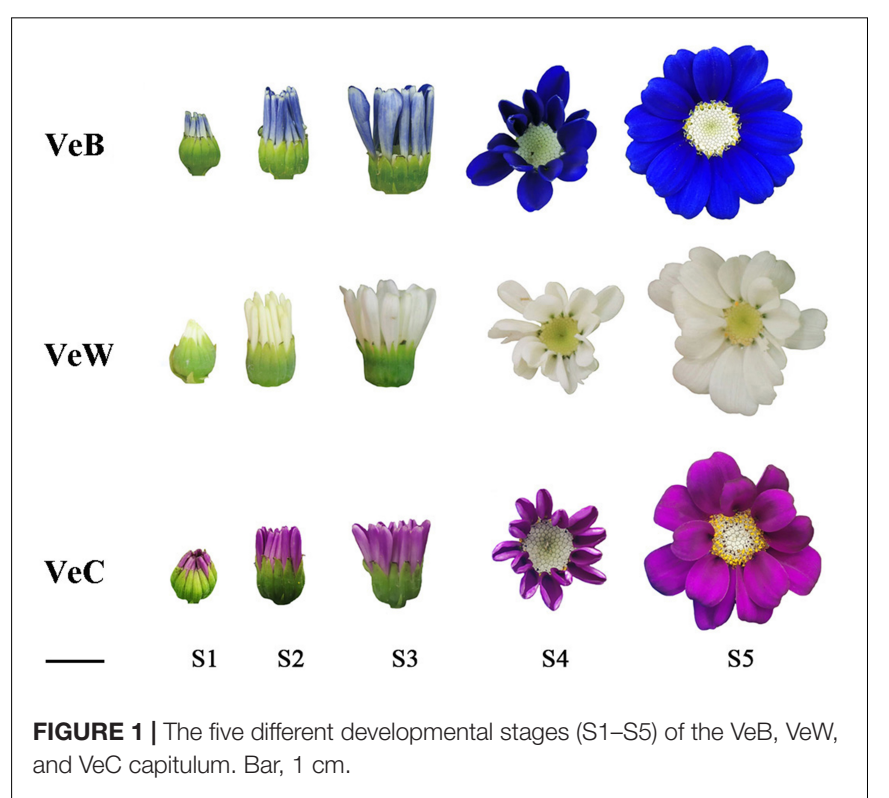

Then, the ray florets of $\mathrm{VeB}, \mathrm{VeC}$, and $\mathrm{VeW}$ at the S1, S2, S3, S4, and S5 stages were sampled for RT-PCR and qRT-PCR analysis.

\section{Identification of Anthocyanins by HPLC-MS/MS}

Anthocyanin accumulation could accumulated in both leaves and ray florets of cineraria. In this study, the leaves and ray florets of $\mathrm{VeB}$ was used to identify anthocyanin components by HPLC-MS/MS analysis, and the ray florets of $\mathrm{VeW}$ and $\mathrm{VeC}$ were used as the control. Anthocyanin extraction and determination were performed as previously described (Sun et al., 2010). A total of $0.1 \mathrm{~g}$ of the sample was finely ground into powder in liquid nitrogen, and then homogenized in $5 \mathrm{ml}$ of anthocyanin extracts [methanol: water: formic acid: trifluoroacetic acid $(70: 27: 2: 1, \mathrm{v} / \mathrm{v} / \mathrm{v} / \mathrm{v})]$, leached at $4^{\circ} \mathrm{C}$ for $24 \mathrm{~h}$ in the dark. The supernatant volume was passed through a $0.22 \mu \mathrm{m}$ filter after centrifugation. The sample was determined by the DIONEX high performance liquid chromatography equipped with a P680 HPLC pump, UltiMate 3000 auto-sampler, Thermostatted Column Compartment-100, and a TSK-GEL ODS-80Ts QA column $(4.6 \mathrm{~mm} \times 150 \mathrm{~mm})$. The loading volume was $20 \mu \mathrm{L}$, mobile phase A was methanol:acetonitrile $=15: 85(\mathrm{v} / \mathrm{v})$, mobile phase $\mathrm{B}$ was formic acid: water $=10: 90(\mathrm{v} / \mathrm{v})$, column temperature was $25^{\circ} \mathrm{C}$, flow rate was $1 \mathrm{~mL} / \mathrm{min}$. The gradient elution was performed as follows (A\%/B\%): 0-20 min, 70\%-47\%/30\%-53\%; 20-40 $\mathrm{min}, 47 \% / 53 \%$; 40-45 $\mathrm{min}, 47 \%-70 \% / 53 \%-30 \%$; $45-$ $60 \mathrm{~min}, 70 \% / 30 \%$. Meanwhile, HPLC-ESI-MS/MS was used to analyze the anthocyanin structure of typical samples by Agilent 1100 LC /MSD Trap VL liquid chromatography-tandem mass spectrometry. The conditions of liquid chromatographic analysis were the same as above. The retention time and peak area of each sample was measured at wavelengths of $520 \mathrm{~nm}$ and in comparison with standards as cyanidin chloride (Sigma). Mass spectrometry analysis of each pigment components was mainly compared with the data of previous study (Sun et al., 2010).

\section{PacBio cDNA Library Construction, Sequencing and Data Analysis}

A transcriptome provides a fast and economical method to systematically characterize gene models for one species without a genome reference. Recently, third-generation fulllength transcriptome sequencing by PacBio SMRT has been widely used in transcriptome sequencing because it provides particularly long reads with high throughput and generates fulllength transcripts (Li et al., 2020; Zhao et al., 2020). Generally, the protocol of transcriptome sequencing was including RNA extraction, transcriptome library construction and sequencing. Total RNA of the mixed samples was extracted using a Quick RNA Isolation Kit (Huayueyang Biotechnology Co. Ltd., Beijing, China). The purity and concentration of the RNA were assessed in a NanoDrop 2000 spectrophotometer. The third-generation full-length transcriptome library construction and sequencing were performed as previously described (Elmore et al., 2020). The main process of constructing a full-length transcriptome library was as follows: First-strand cDNA was synthesized from total RNA using a SMARTer polymerase chain reaction (PCR) 
cDNA Synthesis Kit (Clontech, United States), and then the first-strand cDNA was synthesized with SMARTScribe Reverse Transcriptase. Subsequently, a large amount of double stranded cDNA was produced. Full-length transcriptome library was sequenced by PacBio SMRT. According to the adapter in the sequence, all the original sequences were converted into circularconsensus sequence (CCS), and the sequences were divided into full-length sequences and non-full-length sequences according to whether there were $3^{\prime}$ primers, $5^{\prime}$ primers or PolyA in the CCS. Finally, consensus sequences were calibrated to get the high-quality sequences. All transcript sequences were annotated using the BLASTx alignment $\left(E\right.$-value $\left.<1 \times 10^{-5}\right)$ to the following database: $\mathrm{Nr}$ (National Center for Biotechnology Information non-redundant protein sequences), Pfam (Protein family), UniProtKB/Swiss-port (the UniProt Knowledgebase), COG (Clusters of Orthologous Groups), KOG (euKaryotic Ortholog Groups), GO (Gene Ontology); and KEGG (Kyoto Encyclopedia of Genes and Genomes pathway database). The best-aligning results from these databases were chosen to decide the sequence direction of the unigenes.

\section{Illumina cDNA Library Construction, Sequencing and Data Analysis}

High throughput RNA sequencing (RNA-Seq) via nextgeneration sequencing technologies has been widely used in plants for comparative transcriptomics analysis (Lu et al., 2019; Pu et al., 2020). In this study, the total of RNA of the ray florets at the $\mathrm{S} 1, \mathrm{~S} 2, \mathrm{~S} 4$, and $\mathrm{S} 5$ stages of $\mathrm{VeB}$ and $\mathrm{VeW}$ was extracted using a Quick RNA Isolation Kit (Huayueyang Biotechnology Co. Ltd., Beijing, China). The main process of constructing the next-generation transcriptome library was as follows: magnetic beads with Oligo (dT) were used to enrich Eukaryote RNA, and the mRNA was mixed with the Fragmentation Buffer and fragmented randomly into short fragments. The first strand cDNA was synthesized with random hexamer primers. Subsequently, the second strand cDNA was synthesized using buffer, dNTPs, RNase $H$, and DNA polymerase I. The cDNA were purified with the AMPure XP system. The library was constructed through PCR amplification, and the quality was assessed on the Nanodrop and Agilent 2100 system. Finally, the next-generation transcriptome library was sequenced on Illumina HiSeq Platform. The generated final clean data were then mapped to the above high-quality transcript sequences obtained by third-generation full-length transcriptome sequencing. The expression level of each gene was calculated by using RSEM and converted into fragments per kilobase per million fragments (FPKM) according to the read counts (Li and Dewey, 2011).

\section{Differentially Expressed Transcript Analysis}

To obtain differentially expressed transcripts of ray florets in $\mathrm{VeB}$ and $\mathrm{VeW}$ at the different developmental stages, the differentially expressed genes (DEGs) analysis between B1 and W1, B2 and W2, B4 and W4, B5 and W5 samples were carried out by DESeq package. These DEGs were identified by false discovery rate (False Discovery Rate, FDR) $<0.01$ and a fold change $\geq 2$ and the adjusted $P$-value $<0.05$ were assigned (Anders and Huber, 2010). Differentially expressed genes were classified into functional categories using Blast2GO with the cut-off of $E$-value $<1 \times 10^{-5}$ based on three levels of GO terms: biological processes (BP), molecular function (MF), and cell components (CC) (Conesa et al., 2005). DEGs were also assigned a candidate ko number through searching the KEGG pathway database using the online tool $\mathrm{KAAS}^{1}$ with the cut-off of $E$-value $<1 \times 10^{-5}$ (Moriya et al., 2007). GO and KEGG enrichment analysis of DEGs was performed using the GOseqR packages ${ }^{2}$ and $\mathrm{KOBAS}^{3}$, respectively. Multiple Experiment Viewer software was used for analyzing the expression pattern of DEGs. The Euclidean distance was used to perform K-means cluster analysis on 1,066 differential genes, which differentially expressed in ray florets between $\mathrm{VeB}$ and $\mathrm{VeW}$ at the each developmental stages (S1, S2, S4, and S5), and the clusters positively correlated with the change of anthocyanin content were selected as key candidate clusters (Lin et al., 2018). The R language software package DCGL was used to analyze the genes in the key candidate clusters by WGCNA (Yang et al., 2013; Chen et al., 2016), and the genes related to anthocyanin metabolism were chosen to construct a co-expression network, and Cytoscape software 4 was used to visualize this gene co-expression network (Doncheva et al., 2018).

\section{ScGT/AT Gene Screening and Phylogenetic Analysis}

Genes encoding uridine diphosphate glycosyltransferase, GH1 family members and SCPL acyltransferase are key genes involved in polyacylation modification in blue cineraria. A total of 41 full-length sequences of ScUGTs, 25 full-length sequences of ScGH1s (including 11 full-length sequences of ScAGGTs), and 21 full-length sequences of ScSCPLs were obtained from the full-length transcriptome database in this study and translated using the NCBI Open Reading Frame Finder. Then, sequence alignments were performed using the MUSCLE algorithm in Molecular Evolutionary Genetics Analysis version X (MEGAX) with all Arabidopsis thaliana UGTs, BGLUs, SCPLs, and some amino acid sequences from other plants. Phylogenetic trees of ScUGTs, ScGH1s, and ScSCPLs were subsequently constructed according to the maximum likelihood (ML) estimation method. Tree nodes were evaluated using the bootstrap method for 1,000 replicates (Felsenstein, 1985). Evolutionary distances were computed using the $p$-distance method and expressed in units of amino acid differences per site. All positions containing gaps and missing data were eliminated prior to construction of the phylogenetic trees for the ScUGT, ScGH1, and ScSCPL genes. In addition, the expression patterns of some ScUGTs, ScGH1s, and ScSCPLs, which were clustered in the clades related to flavonoid modification (19 ScUGTs, 11 ScAGGTs, and 3 ScSCPLs), were analysed by RT-PCR.

\footnotetext{
${ }^{1}$ http://www.genome.jp/

${ }^{2}$ http://www.r-project.org/

${ }^{3}$ http://www.kobas.co.uk/

${ }^{4}$ www.cytoscape.org
} 


\section{RT-PCR and qRT-PCR Analysis}

In order to select the key candidate genes which only highly expressed in ray florets of blue cineraria cultivar $\mathrm{VeB}$, the total RNA of ray florets of the five developmental stages in VeW, $\mathrm{VeB}$, and $\mathrm{VeC}$ were extracted using Plant RNA Extraction Kit (HUAYUEYANG Biotechnology, Beijing, China). Then RNA was used to synthesize cDNA for RT-PCR and qRT-PCR with the transcription kit. The specific procedure for RT-PCR refered to the method of previous study (Li et al., 2019). Primers were designed by Primer 5 and the sequences were listed in Supplementary Table 1. The results of RT-PCR were detected by $1 \%$ agarose gel electrophoresis detection. Further expression analysis of highly expressed genes in the ray florets of $\mathrm{VeB}$ selected through RT-PCR were conducted by qRT-PCR. qRTPCR analysis was performed on a CFX96 real-time system (BioRad Laboratories, Hercules, CA, United States) according to the SYBR Premix Ex Taq kit (Japan, Takara) with three replicates. The results of qRT-PCR were read automatically by Bio-Rad CFX Manager and was performed using the $2^{-\Delta} \Delta C T$ method (Livak and Schmittgen, 2001). The primer information is listed in Supplementary Table 2.

\section{Transient Silencing of ScSCPL2 Expression in Blue S. cruentus by VIGS}

As the anthocyanin components of leaves were the same as those in ray florets of blue cineraria cultivar VeB, the VIGS system of cineraria leaves was used to quickly verify the function of the candidate structural gene ScSCPL2, which was performed according to the procedures described by previous study ( $\mathrm{Li}$ et al., 2019). Gene sequences were identified from the full-length transcriptome sequencing database. A 368 bp fragment from the specific nucleotide region of ScSCPL2 was amplified with specific primers (Supplementary Table 3). This PCR product was cloned into pTRV2 to form the pTRV2::ScSCPL2 construct. While pTRV2::ScANS was used as positive control (Li et al., 2019). The pTRV2::ScSCPL2 and pTRV2::ScANS constructs, as well as the pTRV1 which was essential for viral movement (ZieglerGraff et al., 1991) and pTRV2 vectors, were transformed into Agrobacterium tumefaciens strain GV3101 by the freeze-thaw method. The transformed A. tumefaciens lines were grown in LB and resuspended to $\mathrm{OD}_{600}=1.5$ in infiltration buffer. A mixture of A. tumefaciens cultures containing pTRV1 and pTRV2 (or pTRV2::ScSCPL2 or pTRV2::ScANS) was mixed in a $1: 1$ volume ratio before infection.

The infiltration mixture was introduced into 2-leaf stage seedlings by vacuum infiltration (VI). Ten days after the first VI infection, the second infection was carried out through leaf and shoot apical meristem syringe injection (SI), in which the infiltration mixture was introduced into the surfaces until the water-stained area accounted for more than $2 / 3$ of the leaf. After each infection, the seedlings were cultured in the dark at $10^{\circ} \mathrm{C}$ for $2 \mathrm{~d}$ and $15^{\circ} \mathrm{C}$ for $1 \mathrm{~d}$ and then cultured in an illumination incubator at $20^{\circ} \mathrm{C}$ and $60 \%$ relative humidity for $12 \mathrm{~h}$ light $/ 12 \mathrm{~h}$ dark. We observed the seedlings every $3 \mathrm{~d}$ for $30 \mathrm{~d}$.

After the occurrence of the silenced phenotype, silenced tissues were sampled, and PCR was performed to detect the expression of the recombinant plasmid. The changes in anthocyanins in silenced tissues were analysed by HPLC-MS/MS to elucidate the function of ScSCPL2 in polyacylated anthocyanin biosynthesis, and the specific operation procedure was the same as that described above.

\section{RESULTS}

\section{Characteristics of Anthocyanin Components in Leaves and Ray Florets of White, Carmine, and Blue S. cruentus}

Cineraria is a rare plant species with pure and stable blue flowers in nature. In this study, HPLC-MS/MS was used to analyse the pigment composition of $\mathrm{VeB}, \mathrm{VeC}$, and $\mathrm{VeW}$ to explore the chemical basis of the blue colour of ray florets. We found that four different types of anthocyanins, B1, B2, B3, and B4, existed in the ray florets of $\mathrm{VeB}$ (Figure 2C). Two different types of anthocyanins, $\mathrm{C} 1$ and $\mathrm{C} 2$, existed in the ray florets of $\mathrm{VeC}$ (Figure 2B), while no anthocyanins were detected at $520 \mathrm{~nm}$ in VeW (Figure 2A).

MS data showed that the [delphinidin] ${ }^{+}$fragment ion of $\mathrm{m} / \mathrm{z}$ 303 was existed in B1, B2, and B3 peaks. Among them, B3 peak generated fragments at $\mathrm{m} / \mathrm{z} 1,523\left([\mathrm{M}+\mathrm{H}]^{+}\right), \mathrm{m} / \mathrm{z} 1,199$ (loss of one glucosyl and one caffeoyl), m/z 875 (loss of two glucosyls and two caffeoyls), m/z 551 (loss of three glucosyls and three caffeoyls), which were consistent with the described for cinerarin (Goto et al., 1984; Sun et al., 2010). It was identified as Dp3MalG-7d(CafG)-3'CafG, which contained both $3^{\prime}$ and 7 -polyacylated modifications (Figure 2E). B1 peak generated fragments at $\mathrm{m} / \mathrm{z} 875\left([\mathrm{M}+\mathrm{H}]^{+}\right), \mathrm{m} / \mathrm{z} 627$ (loss of one glucosyl and one malonyl), m/z 551 (loss of one glucosyl and one caffeoyl). B2 peak generated fragments at $\mathrm{m} / \mathrm{z} 1,199\left([\mathrm{M}+\mathrm{H}]^{+}\right), \mathrm{m} / \mathrm{z}$ 875 (loss of one glucosyl and one caffeoyl), 551 (loss of two glucosyls and two caffeoyls) (Supplementary Table 4). Based on MS data, UV-vis absorption spectra and published literature (Sun et al., 2010), B1 and B2 peaks were tentatively postulated to be Dp3MalG-3'CafG and Dp3MalG-7CafG-3'CafG, respectively (Figure 2E). Besides, B4 peak contained a [cyanidin] ${ }^{+}$fragment ion of $\mathrm{m} / \mathrm{z}$ 287. It also generated fragments at $\mathrm{m} / \mathrm{z} 1,507$ $\left([\mathrm{M}+\mathrm{H}]^{+}\right), \mathrm{m} / \mathrm{z} 1,183$ (loss of one glucose and one caffeoyl), $\mathrm{m} / \mathrm{z} 859$ (loss of two glucoses and two caffeoyls), m/z 535 (loss of three glucoses and three caffeoyls), which were similar to the fragments of B3 peak. We speculated the chemical structure of B4 was Cy3MalG-7d(CafG)-3'CafG (Figure 2E). Quantitation of these four anthocyanins showed that the content of Dp3MalG$7 \mathrm{~d}(\mathrm{CafG})-3^{\prime} \mathrm{CafG}$ was significantly higher than that of Dp3MalG$3^{\prime} \mathrm{CafG}$ and Dp3MalG-7CafG-3' CafG (Figure 2F). Thus, it was regarded as the main pigment component determining the blue colour of ray florets of cineraria. Furthermore, as C1 and C2 peaks contained the [cyanidin] ${ }^{+}$fragment ion of $\mathrm{m} / \mathrm{z} 287$ based on MS data (Supplementary Table 4), they were regarded as cyanidin derivatives in this study. Quantitation of these two anthocyanins showed that C2 $(221.84 \mu \mathrm{g} / \mathrm{g})$ was the main pigment component determining the carmine colour of ray florets of cineraria (Figure 2F). 
A ${ }_{10}$

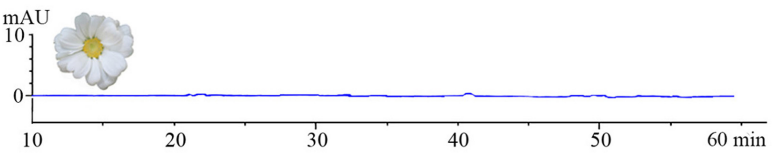

B

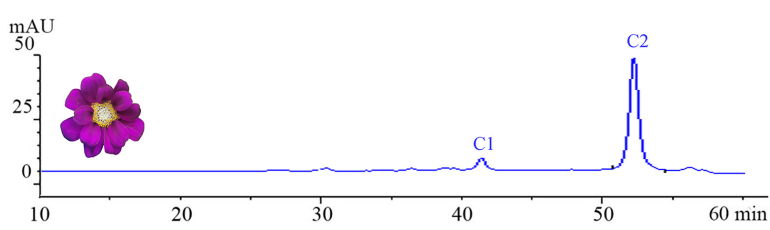

C

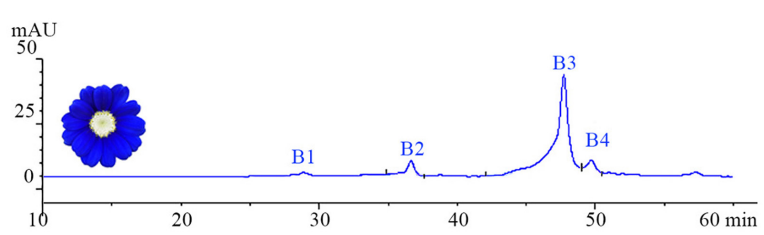

D

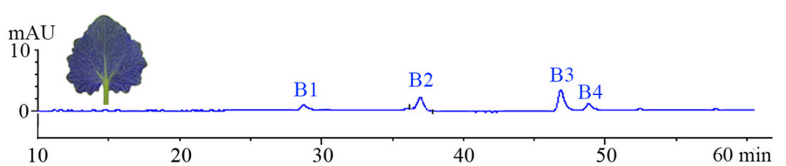

$\mathbf{F}$

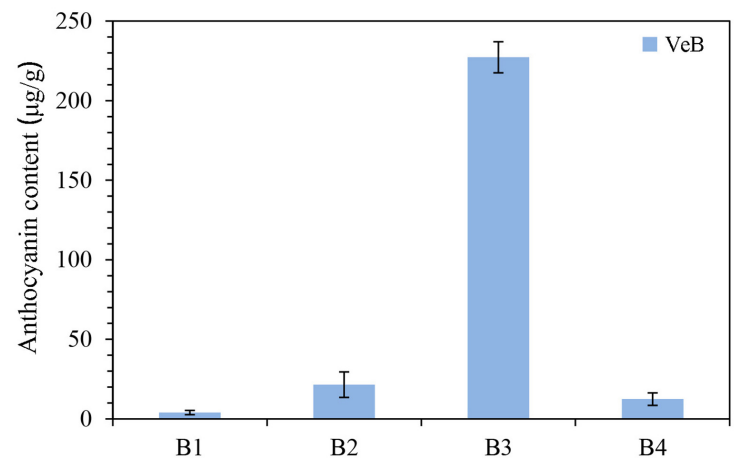

E
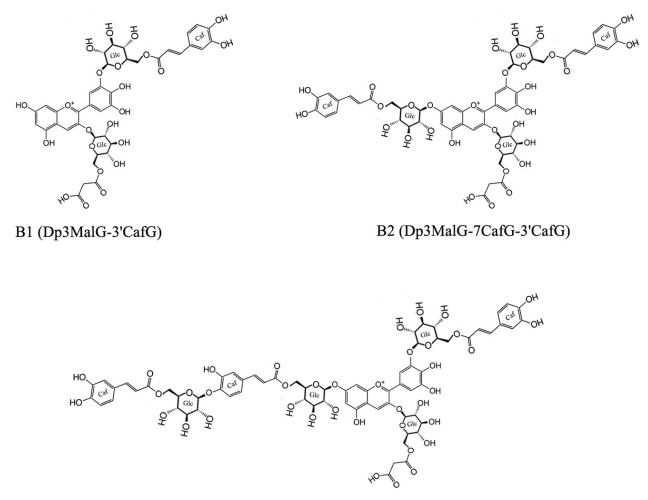

B3 (Dp3MalG-7d(CafG)-3'CafG)
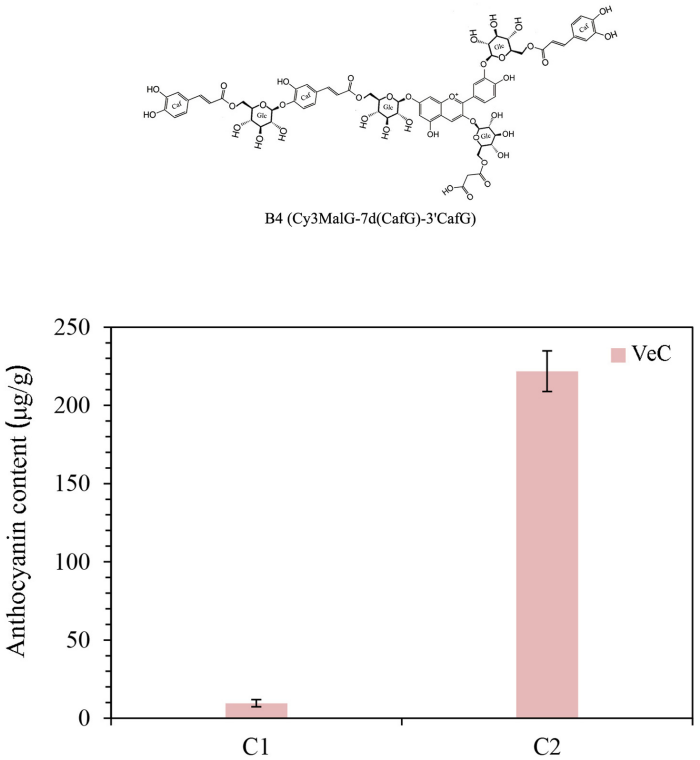

FIGURE 2 | High-performance liquid chromatography (HPLC) analysis (520 nm) of anthocyanin components in the ray florets of VeW (A), VeC (B), and VeB (C). (D) Anthocyanin composition analysis of the leaves of VeB. (E) Hypothetical chemical structures of B1, B2, B3, and B4 peaks. (F) The content of anthocyanins in the ray florets of $\mathrm{VeB}$ and $\mathrm{VeC}$.

In addition, the leaves of $\mathrm{VeB}$ have the same colour as the ray florets. Exploring the consistency of the pigment components in the leaves and the ray florets will help us to perform functional verification of polyacylated genes in blue cineraria leaves by VIGS. HPLC-MS/MS was used to analyse the pigment components of the leaves of $\mathrm{VeB}$, and four peaks also existed (Figure 2D). The UV-vis absorption maxima and protonated molecular data for these four peaks were consistent with those of anthocyanins detected in ray florets, which indicated that the anthocyanin components of leaves were the same as those in ray florets of $\mathrm{VeB}$.

\section{Transcriptome Sequencing and Comparative Transcriptome Analysis}

To identify the key genes for the biosynthesis of polyacylated anthocyanin in blue cineraria, RNA samples of mixed ray florets, leaves and stems were sequenced by PacBio SMRT. A total of $28.1 \mathrm{~Gb}$ clean data were obtained eventually. Based on the parameter of full passes $\geq 1$ and sequence accuracy $>0.9,335$, and 321 CCS were extracted from the original sequences, of which 277,604 were full-length nonchimeric sequences (FLNC), accounting for $82.79 \%$ of the total CCS (Supplementary Figure 1). A total of 32,199 highquality consensus sequences were obtained by clustering the full-length non-chimeric sequences. The low-quality consensus sequences were corrected by the next-generation transcriptome data. Then, they were merged with the highquality consensus sequence to perform de-redundancy analysis, and 19,469 transcript sequences were eventually obtained. These transcript sequences were compared with the databases NR, SwissProt, eggNOG, COG, CO, KOG, KEGG, and Pfam, and a total of 18,882 sequences were annotated (Supplementary Table 5). 
In addition, the ray florets of $\mathrm{VeW}$ and $\mathrm{VeB}$ at the different developmental stages (S1, S2, S4, and S5) were analysed by next-generation sequencing (Figures 3A,B). The non-redundant transcripts measured by full-length transcriptome sequencing were used as a reference for sequence alignment and subsequent analysis. Sequence alignment between clean reads obtained by next-generation sequencing and transcripts through STAR revealed that the average alignment rate of clean reads that can be mapped to the non-redundant transcripts was $69.44 \%$, while the average alignment rate of clean reads that contained the unique position in the transcripts was $47.14 \%$, indicating that the transcriptome data were used efficiently in this study. Then, the transcriptome data of ray florets between $\mathrm{VeW}$ and $\mathrm{VeB}$ at the S1, S2, S4, and S5 stages were compared (W1 vs B1; W2 vs B2; W4 vs B4; and W5 vs B5), and 2,064, 3,295, 3,524, and
2,867 differentially expressed genes (DEGs) were identified and annotated, respectively (Figure 3C). These four groups of DEGs were intersected to screen 1,066 genes that were differentially expressed in VeW and VeB ray florets at the S1, S2, S4, and S5 stages simultaneously (Figure 3D).

Gene Ontology (GO) enrichment analysis was used to define the function of the DEGs. A total of 1,066 DEGs were classified into 18,15 , and 11 categories and assigned to BP, cellular components, and MFs, respectively (Figure 4A). Proteins related to glutathione metabolic process from BP, plastid envelope from cellular component, and glutathione transferase activity from MF were the most enriched GO terms (Figure 4B). To further understand the biological functions of the 1,066 DEGs, KEGG enrichment analysis was used for pathway classification and functional enrichment. The pathways with the most
A
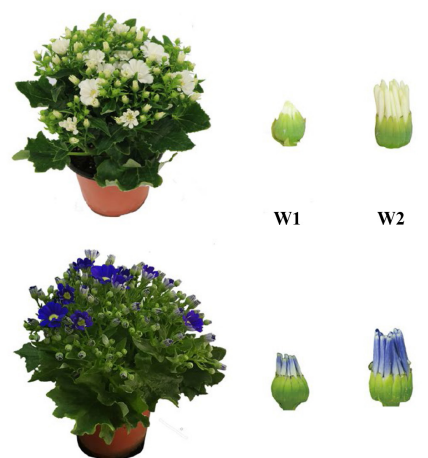

W1

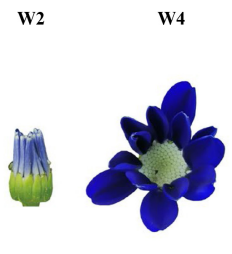

B2

B

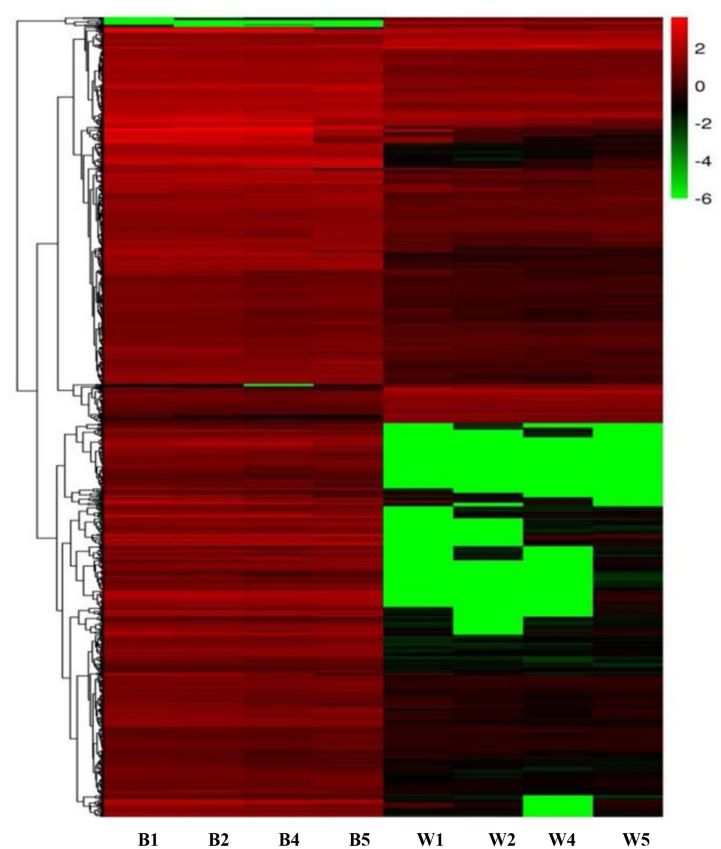

C

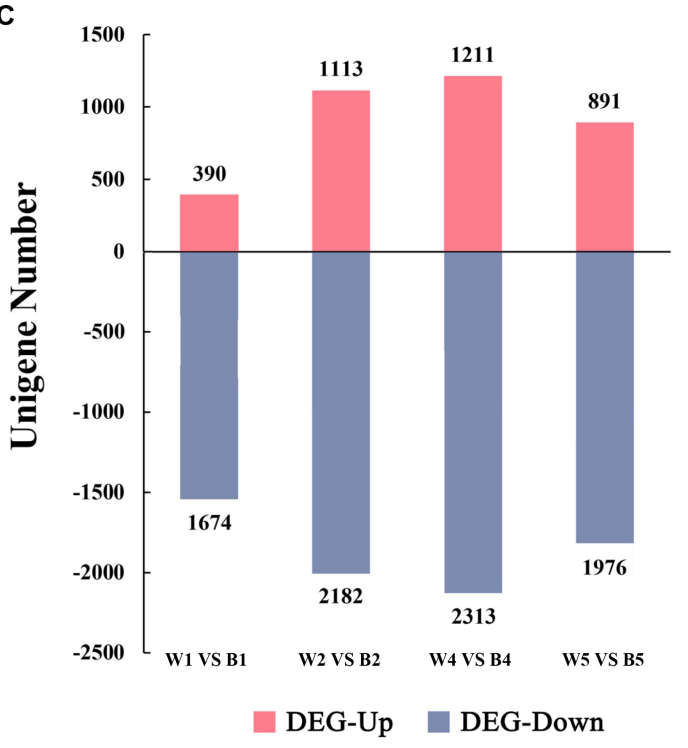

D

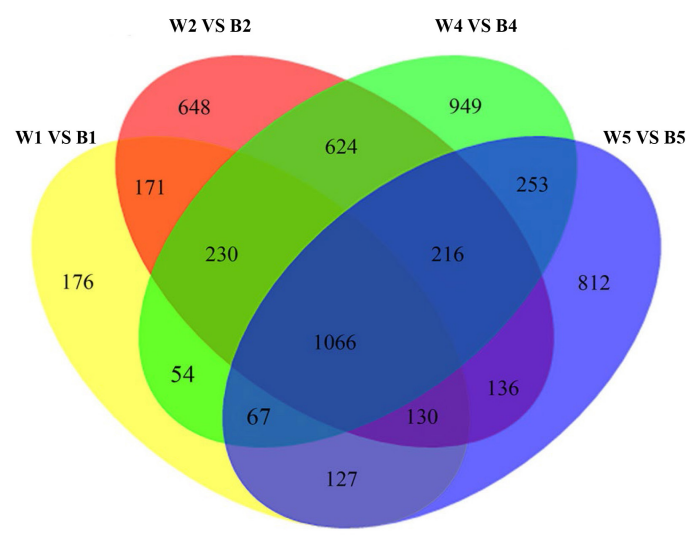

FIGURE 3 | Comparative transcriptomics analysis between the ray florets of VeB and VeW. (A) The ray florets of VeW and VeB at the S1, S2, S4, and S5 stages (renamed W1, W2, W4, W5 and B1, B2, B4, B5, respectively) were used for second-generation sequencing. (B) Heatmap of all gene expression based on the second-generation sequencing data. (C) The number of up-regulated and down-regulated DEGs in comparisons among W1 vs B1, W2 vs B2, W4 vs B4, and W5 vs B5. (D) Venn diagram showing the number of DEGs through paired comparison. 


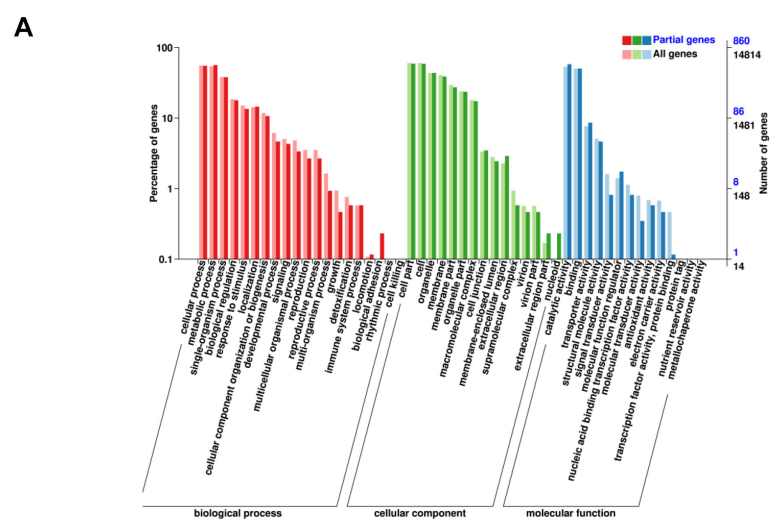

C

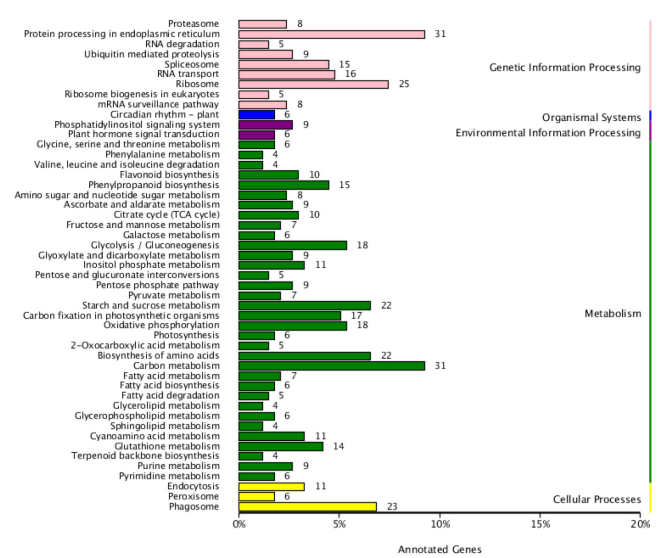

B

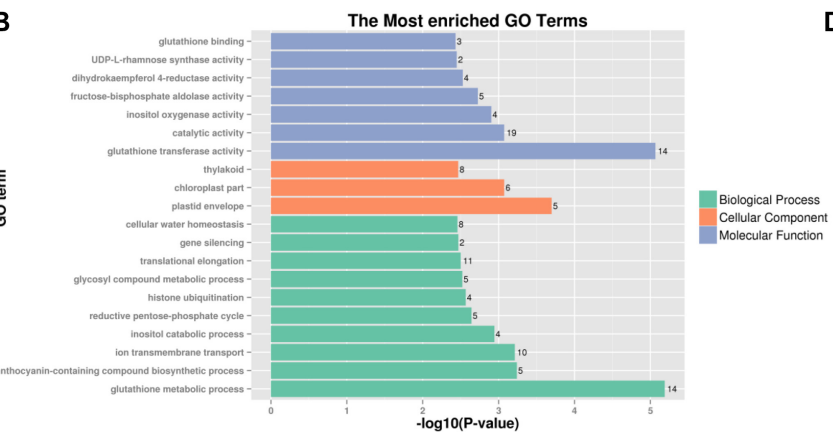

D

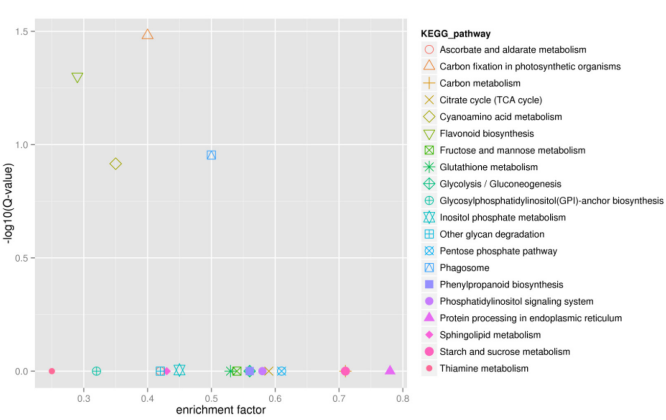

FIGURE 4 | Gene Ontology (GO) and Kyoto Encyclopedia of Genes and Genomes (KEGG) pathway functional classification and enrichment analysis of the 1,066 differentially expressed genes (DEGs) identified from Venn diagram. (A) Functional classification of DEGs based on GO categorization. (B) GO enrichment analysis of the DEGs. (C) Pathway assignment based on the KEGG classification metabolism categories. (D) KEGG pathway enrichment analysis of the DEGs.

DEG enrichment were carbon metabolism (ko01200), protein processing in endoplasmic reticulum (ko04141), and ribosome (ko03010). In addition, some DEGs were also enriched in flavonoid biosynthesis (ko00941) (Figures 4C,D).

A total of 1,066 differentially expressed transcripts were divided into 11 clusters by K-means cluster analysis based on the expression profiles of these genes. The results revealed that the expression modes of genes in cluster 3 , cluster 6 , and cluster 8 appeared to be significantly positively correlated with the changes in anthocyanin content in $\mathrm{VeW}$ and $\mathrm{VeB}$ (Figure 5). A large number of structural genes and transcription factors associated with anthocyanin metabolism were found from these three clusters, including structural genes (ScCHSs, ScF3Hs, ScF3' $5^{\prime} \mathrm{H}$, ScDFR, ScANS, ScMT, and ScGSTs), transcription factors (ScMYBs and ScbHLHs) and modified genes (ScUGTs, ScGH1s, and ScSCPLs), which may be involved in the polyacylation modification of blue cineraria (Table $\mathbf{1}$ ).

\section{Identification of ScGTs/ATs Involved in Polyacylated Anthocyanin Biosynthesis}

The alternation of glucosylation and caffeoylation catalysed by glucosyltransferase UGTs/GH1s and caffeoyl transferase SCPLs, respectively, at the $3^{\prime}$ or 7-position of anthocyanins ultimately leads to the formation of polyacylated cinerarin, which is crucial to blue ray floret coloration. To identify all genes encoding glycolyltransferase and acyltransferase that may be involved in anthocyanin polyacylation modification, we searched these three groups of structural genes from the third-generation transcriptome database and obtained a total of 41 full-length sequences of UGTs, 25 full-length sequences of GH1s (including 11 full-length sequences of AGGTs, which belong to $G H 1$, catalyse the glycosylation of anthocyanins with acyl-glucoses as glucosyl donors), and 21 full-length sequences of SCPLs.

Phylogenetic analysis of 41 ScUGTs revealed that 19 of them were clustered in four clades related to flavonoid glycosylation. ScUGT15, ScUGT17, ScUGT23, ScUGT29, ScUGT31, ScUGT32, and ScUGT39 were clustered in Clade I with the genes encoding anthocyanin 3-O-glucosyltransferase in Arabidopsis thaliana, Vitis vinifera, and Gentiana triflora (Figure 6A), which may be involved in modifying the glycosylation of the 3-O-position of anthocyanins in cineraria. ScUGT1 and ScUGT3 were clustered with the genes identified from Perilla frutescens, Verbena hybrida, P. hybrid, and G. triflora in Clade II (Figure 6A), which were related to the glycosylation of the 5-O-position of anthocyanins. These genes were also clustered with $D g p H B A G T$, which is involved in the synthesis of $p$-hydroxybenzoyl-glucose (pHBG), which acts as a zwitter donor in acylation and glucosylation in D. grandiflorum (Nishizaki et al., 2014). ScUGT4, ScUGT14, ScUGT25, ScUGT27, and ScUGT28 were clustered with $G t 3^{\prime} G T$ and SbUF7GlcT in Clade IIIa (Figure 6A), 

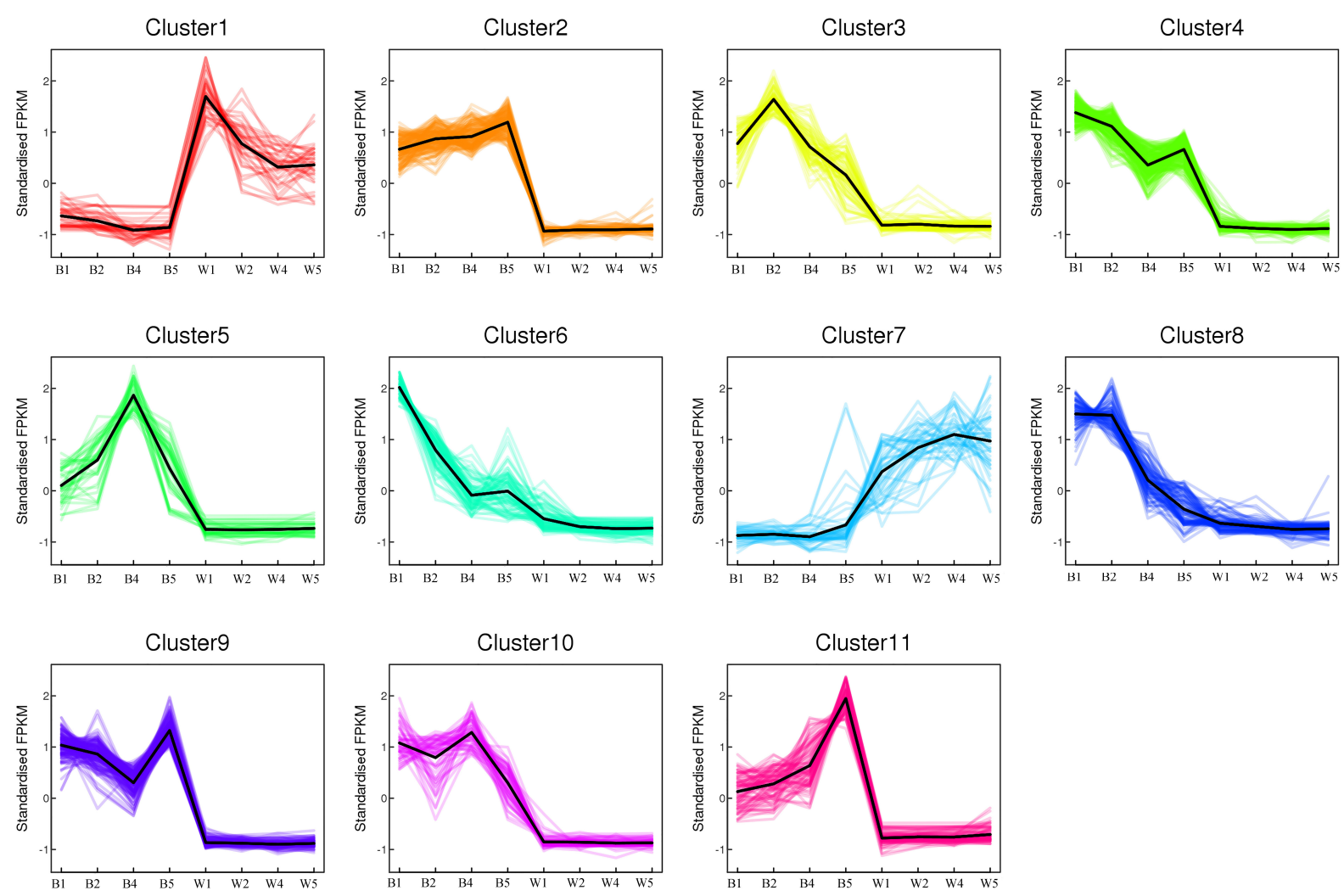

FIGURE 5 | K-means analysis of 1,066 DEGs identified from Venn diagram. A total of 11 clusters were identified based on the expression profiles of these genes.

which might be involved in the glycosylation of the $3^{\prime}-\mathrm{O}-$ position of cinerarin. In addition, ScUGT13, ScUGT19, ScUGT21, ScUGT22, and ScUGT24 were clustered with VPUF7GAT, PfUF7GAT, and AmUF7GAT in Clade IIIb (Figure 6A), which specifically catalysed the glycosylation of the 7-O-position of flavones with UDP-glucuronic acid as the glycosyl donor (Ono et al., 2010). Phylogenetic analysis of 25 ScGH1s showed that ScAGGT2, ScAGGT3, ScAGGT5, ScAGGT9, ScAGGT10, ScAGGT11, ScAGGT12, ScAGGT13, ScAGGT23, ScAGGT31, and ScAGGT38 were clustered with AtBGLU10, DgAA7GT, DcAA5GT, and AaAA7GT (Figure 6B), which catalysed the glycosylation of the 5- or 7-O-position of anthocyanins in Clade I, which indicated that these ScAGGTs might be involved in the glycosylation of the 7-O-position of cinerarin.

In addition, phylogenetic analysis of 21 ScSCPLs showed that ScSCPL2, ScSCPL8, and ScSCPL18 were clustered in Clade Ia with genes encoding flavonoid acyltransferase in C. ternatea, A. thaliana, and D. grandiflorum (Figure 6C) (Noda et al., 2006; Fraser et al., 2007; Nishizaki et al., 2013; Shi and Xie, 2014). ScSCPL1, ScSCPL9, ScSCPL13, and ScSCPL16 were clustered in Clade Ib (Figure 6C), and these genes might be related to the plant wound response (Moura et al., 2001). ScSCPL3, ScSCPL4, ScSCPL5, ScSCPL6, ScSCPL7, ScSCPL17, and ScSCPL19 were clustered in Clade II (Figure 6C), of which the members play an important role in plant growth and development, such as brassinolide signalling and seed germination (Li et al., 2001).
ScSCPL11, ScSCPL12, ScSCPL14, and ScSCPL21 were clustered in Clade III (Figure 6C), however, the function of this clade is not clear.

The expression patterns of some ScUGTs, ScAGGTs, and ScSCPLs, which were clustered in the clades related to flavonoid modification (19 ScUGTs, 11 ScAGGTs, and 3 ScSCPLS), were analysed by RT-PCR. The results showed that ScUGT23 and ScUGT39, belonging to Clade I UGTs, were highly expressed in the ray florets of $\mathrm{VeB}$ and at a high expression level in $\mathrm{VeC}$ but almost not expressed in $\mathrm{VeW}$ (Figure 6D), which indicated that ScUGT23 and ScUGT39 might be involved in the primary glycosylation of 3-O-position of anthocyanin in cineraria. ScUGT1 clustered with DgpHBAGT in Clade II, was highly expressed in $\mathrm{VeB}$ and might be involved in the synthesis of caffeoylglucose. ScUGT4 clustered with $G t 3^{\prime} G T$ in Clade IIIa was highly expressed in $\mathrm{VeB}$ but almost not expressed in $\mathrm{VeW}$ and $\mathrm{VeC}$ (Figure 6D), which indicated that it might be involved in the glycosylation of 3'-O-position of cinerarin. RT-PCR analysis also revealed that ScAGGT11 and ScAGGT12 clustered with $D g A A 7 G T$ and AaAA7GT in Clade I highly expressed in VeB but slightly expressed in $\mathrm{VeC}$ and $\mathrm{VeW}$ (Figure 6D), which indicated that ScAGGT11 and ScAGGT12 might be involved in the glycosylation of the 7-O-position of cinerarin.

In terms of acylation modification of cineraria, SCSCPL2, which belonged to Clade Ia, was highly expressed in the ray florets of $\mathrm{VeB}$, slightly expressed in $\mathrm{VeC}$ at the $\mathrm{S} 1$ stage, and almost not 
TABLE 1 | Stuctural genes and transcription factors associated with anthocyanin metabolism in 3, 6, and 8 clusters.

\begin{tabular}{|c|c|c|c|c|c|c|c|c|c|c|c|}
\hline Cluster & \#ID & B1 FPKM & B2 FPKM & B3 FPKM & B4_FPKM & W1 FPKM & W2_FPKM & W3_FPKM & W4_FPKM & nr_annotation & Abbreviation \\
\hline \multirow[t]{6}{*}{ cluster3 } & F01_transcript/20170 & 412.61 & $1,056.81$ & 378.29 & 0.08 & 79.96 & 183.43 & 3.62 & 4.81 & $\begin{array}{l}\text { RecName: Full = Chalcone synthase; } \\
\text { AltName: Full = Naringenin-chalcone } \\
\text { synthase [Callistephus chinensis] }\end{array}$ & $\mathrm{CHS}$ \\
\hline & F01_transcript/20876 & 49.10 & 61.44 & 45.76 & 49.70 & 19.53 & 20.26 & 18.90 & 22.18 & $\begin{array}{l}\text { Bifunctional dihydroflavonol } \\
\text { 4-reductase/flavanone 4-reductase } \\
\text { isoform } 1 \text { [Theobroma cacao] }\end{array}$ & $D F R$ \\
\hline & F01_transcript/17805 & $3,185.37$ & $3,734.92$ & 2,949.63 & 434.19 & 56.43 & 21.04 & 21.82 & 23.60 & $\begin{array}{l}\text { hypothetical protein [Delphinium } \\
\text { grandiflorum] }\end{array}$ & ScGH1-9 \\
\hline & F01_transcript/17421 & 47.10 & 71.15 & 32.83 & 41.27 & 0.23 & 0.13 & 0.14 & 0.37 & $\begin{array}{l}\text { serine carboxypeptidase precursor } \\
\text { [Matricaria chamomilla] }\end{array}$ & SCSCPL3 \\
\hline & F01_transcript/17893 & 93.60 & 128.50 & 66.00 & 44.17 & 0.04 & 0.07 & 0.05 & 0.25 & $\begin{array}{l}\text { PREDICTED: UDP-glucose } \\
\text { 6-dehydrogenase } 3 \text { [Nicotiana sylvestris] }\end{array}$ & ScUGT2 \\
\hline & F01_transcript/14067 & 118.81 & 184.96 & 118.06 & 98.38 & 7.95 & 17.71 & 17.80 & 8.08 & $\begin{array}{l}\text { PREDICTED: probable methyltransferase } \\
\text { PMT15 [Nicotiana sylvestris] }\end{array}$ & MT \\
\hline \multirow[t]{9}{*}{ cluster6 } & F01_transcript/15677 & 9.59 & 5.74 & 3.75 & 2.63 & 0.42 & 0.35 & 0.37 & 0.35 & $\begin{array}{l}\text { Putative methyltransferase family protein } \\
\text { [Solanum demissum] }\end{array}$ & MT \\
\hline & F01_transcript/14664 & 14.99 & 4.50 & 5.99 & 1.23 & 0.00 & 0.00 & 0.00 & 0.00 & $\begin{array}{l}\text { PREDICTED: probable methyltransferase } \\
\text { PMT19 [Ne/umbo nucifera] }\end{array}$ & MT \\
\hline & F01_transcript/14764 & 101.69 & 37.18 & 7.36 & 1.01 & 2.31 & 0.09 & 0.00 & 0.05 & $\begin{array}{l}\text { PREDICTED: beta-glucosidase 11-like } \\
\text { isoform X1 [Vitis vinifera] }\end{array}$ & ScGH1-2 \\
\hline & F01_transcript/14853 & 195.30 & 78.52 & 27.49 & 1.25 & 3.35 & 0.26 & 0.00 & 0.00 & $\begin{array}{l}\text { unnamed protein product [Coffea } \\
\text { canephora] }\end{array}$ & ScGH1-3 \\
\hline & F01_transcript/16506 & 12.23 & 8.72 & 2.62 & 2.80 & 2.53 & 0.74 & 0.25 & 0.44 & $\begin{array}{l}\text { PREDICTED: glucan } \\
\text { endo-1,3-beta-glucosidase } 1 \text { [Nicotiana } \\
\text { sylvestris] }\end{array}$ & ScGH1-4 \\
\hline & F01_transcript/18027 & 335.20 & 281.57 & 102.18 & 5.34 & 1.98 & 0.48 & 0.00 & 0.35 & $\begin{array}{l}\text { hypothetical protein [Delphinium } \\
\text { grandiflorum] }\end{array}$ & ScGH1-10 \\
\hline & F01_transcript/29185 & 70.47 & 42.36 & 21.38 & 16.51 & 1.21 & 0.28 & 0.08 & 0.07 & $\begin{array}{l}\text { PREDICTED: ultraviolet-B receptor UVR8 } \\
\text { [Populus euphratica] }\end{array}$ & UVR8 \\
\hline & F01_transcript/26228 & 177.84 & 137.87 & 52.74 & 3.26 & 189.47 & 83.97 & 22.78 & 40.49 & $\begin{array}{l}\text { PREDICTED: myb-related protein } \\
\text { 308-like [Populus euphratica] }\end{array}$ & SCMYB1 \\
\hline & F01_transcript/25625 & 826.38 & 618.56 & 283.88 & 36.04 & 754.49 & 212.47 & 24.92 & 148.17 & $\begin{array}{l}\text { transcription factor GbMYB1 [Gynura } \\
\text { bicolor] }\end{array}$ & ScMYB3 \\
\hline \multirow[t]{7}{*}{ cluster8 } & F01_transcript/8489 & 100.91 & 72.29 & 26.33 & 2.14 & 2.48 & 0.82 & 0.83 & 0.34 & $\begin{array}{l}\text { dihydroflavonol 4-reductase [Pericallis } \\
\text { cruenta] }\end{array}$ & $D F R$ \\
\hline & F01_transcript/22631 & 602.84 & 405.08 & 218.02 & 3.77 & 19.40 & 0.53 & 0.10 & 0.02 & $\begin{array}{l}\text { anthocyanidin synthase [Pericallis } \\
\text { cruenta] }\end{array}$ & ANS \\
\hline & F01_transcript/3423 & 63.99 & 52.63 & 18.67 & 0.29 & 17.90 & 13.28 & 0.76 & 4.21 & flavanone 3-hydroxylase [Gynura bicolor] & $\mathrm{F} 3 \mathrm{H}$ \\
\hline & F01_transcript/21258 & 1,353.22 & $1,239.51$ & 919.56 & 39.43 & 344.68 & 232.82 & 38.25 & 114.85 & flavanone 3-hydroxylase [Gynura bicolor] & $\mathrm{F} 3 \mathrm{H}$ \\
\hline & F01_transcript/10531 & $1,004.11$ & 908.97 & 348.96 & 11.52 & 71.63 & 7.37 & 0.33 & 1.58 & flavanone 3-hydroxylase [Gynura bicolor] & F3H \\
\hline & F01_transcript/21783 & $1,389.48$ & $1,093.78$ & 453.99 & 55.81 & 45.46 & 10.89 & 17.24 & 5.74 & $\begin{array}{l}\text { dihydroflavonol reductase [Gynura } \\
\text { bicolor] }\end{array}$ & $D F R$ \\
\hline & F01_transcript/21611 & $1,500.68$ & $1,171.75$ & 668.33 & 64.66 & 1.86 & 1.16 & 3.78 & 0.26 & $\begin{array}{l}\text { dihydroflavonol reductase [Gynura } \\
\text { bicolor] }\end{array}$ & $D F R$ \\
\hline
\end{tabular}




\begin{tabular}{|c|c|c|c|c|c|c|c|c|c|c|c|}
\hline Cluster & $\# I D$ & B1 FPKM & B2 FPKM & B3 FPKM & B4_FPKM & W1 FPKM & W2_FPKM & W3_FPKM & W4_FPKM & nr_annotation & Abbreviation \\
\hline & F01_transcript/23062 & $1,478.20$ & $1,228.58$ & 726.19 & 51.23 & 61.72 & 15.91 & 28.77 & 7.96 & $\begin{array}{l}\text { dihydroflavonol reductase [Gynura } \\
\text { bicolor] }\end{array}$ & DFR \\
\hline & F01_transcript/18084 & $3,611.16$ & 3,329.84 & $2,576.61$ & 119.82 & 85.51 & 4.41 & 1.83 & 0.94 & $\begin{array}{l}\text { PREDICTED: serine } \\
\text { carboxypeptidase-like[Nicotiana } \\
\text { tomentosiformis] }\end{array}$ & ScSCPL2 \\
\hline & F01_transcript/17059 & $4,165.92$ & $2,916.19$ & $1,632.97$ & 90.84 & 104.27 & 3.44 & 1.34 & 1.56 & $\begin{array}{l}\text { glycoside hydrolase family } 1 \text { [Artemisia } \\
\text { annua] }\end{array}$ & ScGH1-5 \\
\hline & F01_transcript/17114 & 295.91 & 105.11 & 33.61 & 3.82 & 9.74 & 0.36 & 0.04 & 0.00 & $\begin{array}{l}\text { glycoside hydrolase family } 1 \text { [Artemisia } \\
\text { annua] }\end{array}$ & ScGH1-6 \\
\hline & F01_transcript/17805 & 55.97 & 67.27 & 54.70 & 7.74 & 0.06 & 0.00 & 0.00 & 0.31 & $\begin{array}{l}\text { beta-glucosidase } 11 \text { isoform } \times 4 \\
\text { [Helianthus annuus] }\end{array}$ & ScGH1-11 \\
\hline & F01_transcript/18352 & 380.81 & 536.20 & 749.78 & 111.22 & 0.18 & 0.00 & 0.46 & 0.00 & $\begin{array}{l}\text { beta-glucosidase } 11 \text {-like isoform X3 } \\
\text { [Lactuca sativa] }\end{array}$ & ScGH1-12 \\
\hline & F01_transcript/7187 & 74.91 & 53.76 & 20.34 & 2.03 & 0.38 & 0.07 & 0.02 & 0.14 & $\begin{array}{l}\text { PREDICTED: beta-glucosidase 22-like } \\
\text { [Elaeis guineensis] }\end{array}$ & ScGH1-13 \\
\hline & F01_transcript/17811 & 847.47 & 837.09 & 337.54 & 6.91 & 20.62 & 0.85 & 0.21 & 0.95 & $\begin{array}{l}\text { PREDICTED: crocetin } \\
\text { glucosyltransferase, chloroplastic-like } \\
\text { [Vitis vinifera] }\end{array}$ & ScUGT1 \\
\hline & F01_transcript/17925 & 688.85 & 704.08 & 386.07 & 12.27 & 18.22 & 0.45 & 0.28 & 0.47 & $\begin{array}{l}\text { PREDICTED: crocetin } \\
\text { glucosyltransferase, chloroplastic-like } \\
\text { [Vitis vinifera] }\end{array}$ & ScUGT3 \\
\hline & F01_transcript/20913 & 71.08 & 69.76 & 35.73 & 31.14 & 1.94 & 1.88 & 3.81 & 1.46 & $\begin{array}{l}\text { PREDICTED: probable } \\
\text { beta-1,3-galactosyltransferase 10-like } \\
\text { [Glycine max] }\end{array}$ & ScUGT5 \\
\hline & F01_transcript/18974 & 7.26 & 8.09 & 19.95 & 72.64 & 0.28 & 0.15 & 0.12 & 0.41 & $\begin{array}{l}\text { UDP-glycosyltransferase 73A17 [Camellia } \\
\text { sinensis] }\end{array}$ & ScUGT4 \\
\hline & F01_transcript/16894 & 354.30 & 231.58 & 156.77 & 7.14 & 35.22 & 1.41 & 0.15 & 0.20 & bHLH transcription factor [Dahlia pinnata] & $b H L H$ \\
\hline & F01_transcript/13375 & 202.60 & 166.73 & 66.03 & 10.36 & 5.47 & 1.44 & 1.00 & 2.12 & bHLH transcription factor [Dahlia pinnata] & $b H L H$ \\
\hline & F01_transcript/23148 & 21.02 & 31.99 & 4.09 & 4.59 & 0.24 & 0.07 & 0.11 & 0.12 & $\begin{array}{l}\text { PREDICTED: transcription factor bHLH79 } \\
\text { [Prunus mume] }\end{array}$ & $b H L H$ \\
\hline & F01_transcript/2735 & 60.56 & 54.51 & 19.62 & 1.72 & 5.54 & 0.25 & 0.03 & 0.04 & bHLH transcription factor [Dahlia pinnata] & $b H L H$ \\
\hline & F01_transcript/6748 & 38.62 & 35.73 & 13.08 & 1.71 & 0.57 & 0.29 & 0.20 & 0.35 & bHLH transcription factor [Dahlia pinnata] & $b H L H$ \\
\hline & F01_transcript/1904 & 16.61 & 16.91 & 5.32 & 0.42 & 1.54 & 0.13 & 0.00 & 0.00 & bHLH transcription factor [Dahlia pinnata] & $b H L H$ \\
\hline & F01_transcript/27268 & 12.13 & 40.92 & 16.35 & 0.25 & 1.60 & 0.10 & 0.62 & 1.01 & $\begin{array}{l}\text { Transcription repressor MYB4 -like } \\
\text { protein [Gossypium arboreum] }\end{array}$ & SCMYB2 \\
\hline & F01_transcript/30445 & 980.63 & 349.26 & 71.79 & 0.29 & 1.43 & 0.14 & 0.14 & 0.18 & $\begin{array}{l}\text { PREDICTED: MYB-like transcription } \\
\text { factor ETC1 [Eucalyptus grandis] }\end{array}$ & SCMYB4 \\
\hline & F01_transcript/18873 & 779.80 & 503.07 & 156.28 & 0.03 & 23.29 & 0.05 & 0.02 & 0.08 & $\begin{array}{l}\text { flavonoid-3',5'-hydroxylase [Pericallis } \\
\text { cruenta] }\end{array}$ & $F^{\prime} 5^{\prime} H$ \\
\hline & F01_transcript/24911 & 151.57 & 95.33 & 58.26 & 2.78 & 0.68 & 0.04 & 0.00 & 0.00 & glutathione S-transferase [Dahlia pinnata] & GST \\
\hline & F01_transcript/25545 & $3,672.06$ & $4,691.52$ & $2,440.66$ & 195.28 & 182.62 & 63.23 & 22.85 & 13.72 & $\begin{array}{l}\text { PREDICTED: glutathione } \\
\text { S-transferase-like [Eucalyptus grandis] }\end{array}$ & GST \\
\hline & F01_transcript/26648 & $1,187.26$ & $1,125.50$ & $1,074.77$ & 62.14 & 72.10 & 2.37 & 0.53 & 0.08 & glutathione S-transferase [Dahlia pinnata] & GST \\
\hline & F01_transcript/26883 & $1,013.47$ & 628.40 & 452.50 & 21.19 & 31.43 & 1.48 & 0.19 & 0.42 & glutathione S-transferase [Dahlia pinnata] & GST \\
\hline
\end{tabular}

F01_transcript/26883

$1,187.26$
$1,013.47$

628.40

62.14
21.19

31.43

0.19 glutathione S-transferase [Dahlia pinnata] 
A

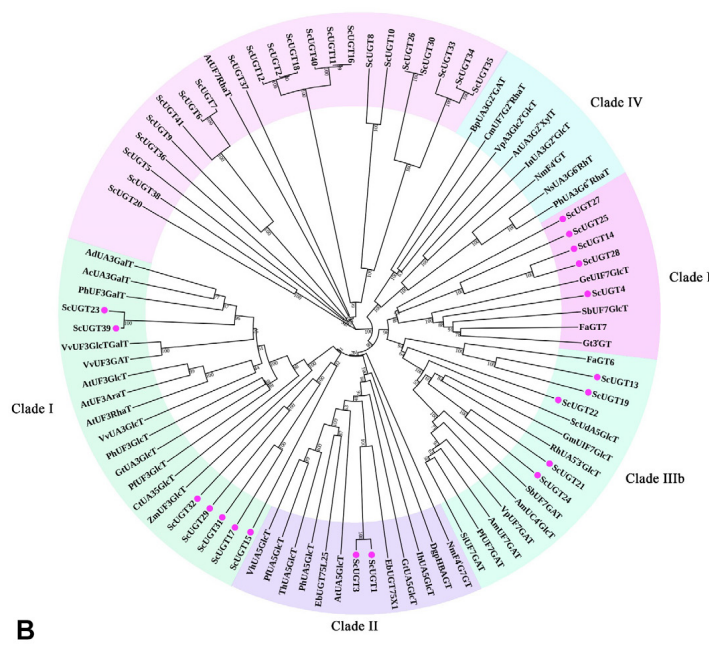

B
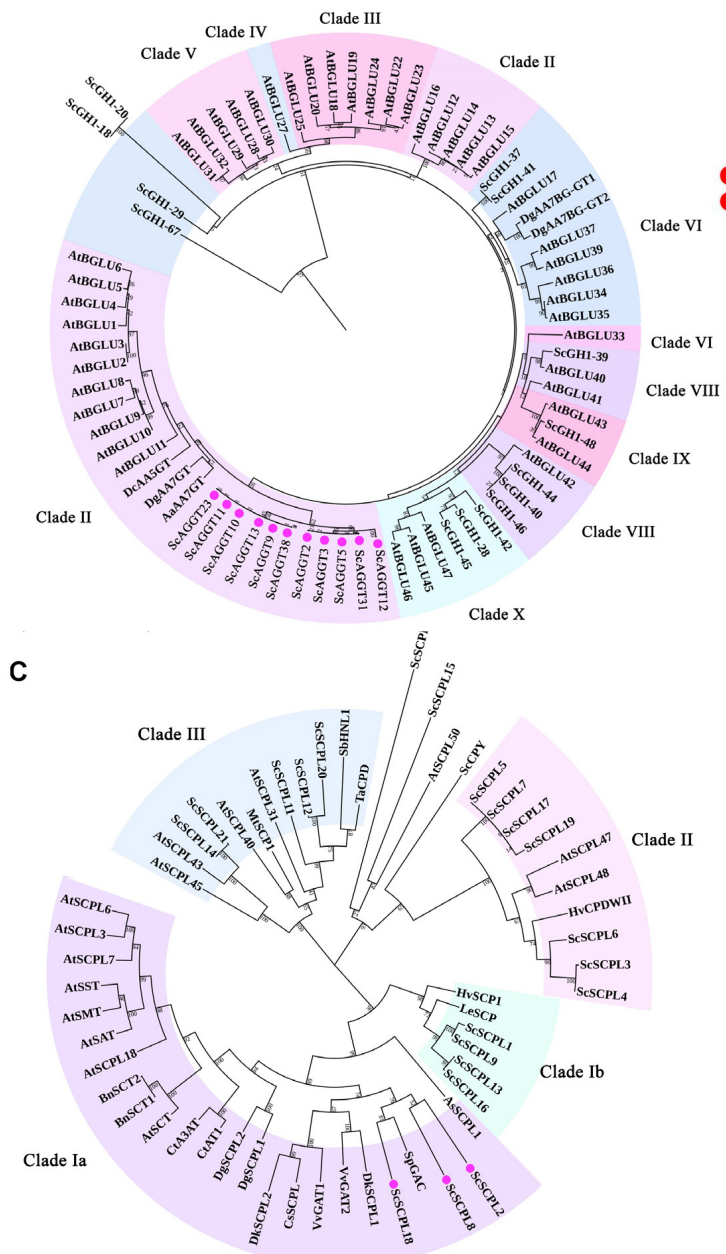

D

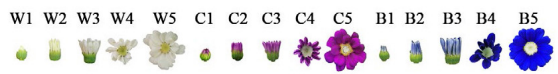

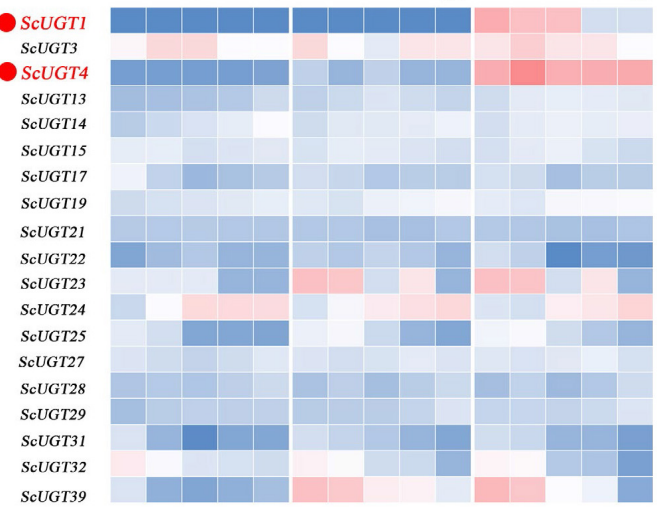

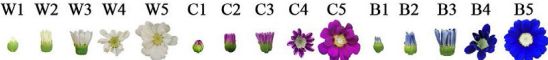

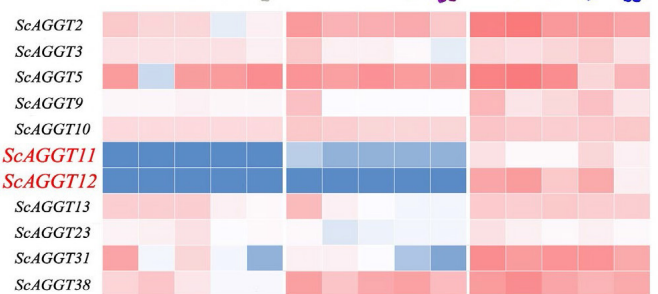

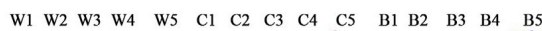

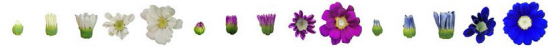

SCSCPL

SeSCPLS

ScSCPL18

E
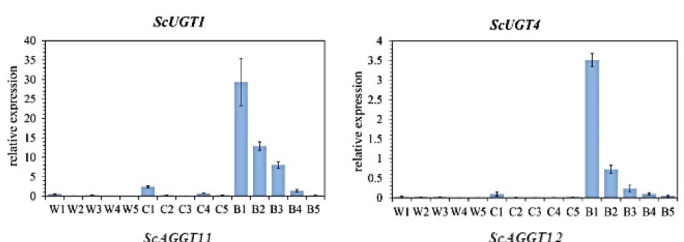

SCAGGI12
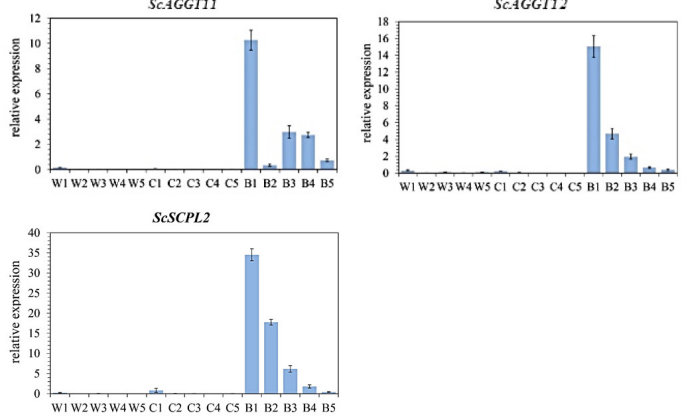

FIGURE 6 | Identification of SCUGTS/SCGH1S/SCSCPLS involved in polyacylated anthocyanin biosynthesis by phylogenetic analysis and gene expression pattern analysis. Amino acid sequences of ScUGTS (A), ScGH1s (B), ScSCPLS (C), and all arabidopsis UGTs/BGLUs/SCPLs and some sequences from other plants were used to build three phylogenetic trees based on maximum likelihood. (D) Heatmap analysis based on the RT-PCR data was performed to screened out the candidate key genes (SCUGTS/SCAGGTS/SCSCPLS) encoding glycolyltransferase and acyltransferase that might be involved in the polyacylation modification of anthocyanins. (E) The expression profiles of ScUGT1, ScUGT4, SCAGGT11, SCAGGT12, and SCSCPL2 were analyzed by qRT-PCR. The data were the mean \pm SD from three biological replicates. 
expressed in $\mathrm{VeC}$ at the other developmental stages or in $\mathrm{VeW}$. Furthermore, the expression levels of ScSCPL8 and ScSCPL18, which also belonged to Clade Ia, were not significantly different among $\mathrm{VeB}, \mathrm{VeC}$, and $\mathrm{VeW}$ (Figure 6D). These results indicated that ScSCPL2 might be involved in the acylation of blue cineraria. qRT-PCR verified the above results (Figure $6 \mathrm{E}$ ). In addition, we found that these candidate modified genes (ScUGT1, ScUGT4, ScAAGT11, ScAAGT12, and ScSCPL2) all existed in cluster 8 of DEGs. Sequence analysis of ScUGT1 and ScUGT4 showed that their amino acid sequences shared the common domain of a PSPG box (putative secondary product glycosyltransferase) in the $C$-terminal region (Figure 7A). Meanwhile, two catalytically active glutamate residue sequences of ScAAGT11 and ScAAGT12 (Figure 7B) as well as putative substrate binding and catalytic regions of ScSCPL2 were also identified and marked (Figure 7C).

\section{Transient Silencing of ScSCPL2 Expression by VIGS in Blue Cineraria}

As the anthocyanin components of leaves were the same as those in ray florets of VeB, the VIGS system of cineraria leaves was used to quickly verify the function of the candidate structural gene ScSCPL2. The vectors, including pTRV1, pTRV2, pTRV2::ScANS and pTRV2::ScSCPL2, were transformed into A. tumefaciens strain GV3101 by the freeze-thaw method. Then, the infiltration mixture containing pTRV1 and pTRV2 (or pTRV2::ScANS or pTRV2::ScSCPL2) was introduced into 2-leaf stage seedlings by VI. Among them, pTRV2::ScANS was used as a positive control to show the feasibility of this VIGS system (Li et al., 2019). The leaves of blue cineraria infiltrated with pTRV2::ScSCPL2 exhibited a function-loss phenotype, whose colour turned pink, at 30 days post-infiltration. No leaves infiltrated with pTRV2 (regarded as a negative control) eventually exhibited a functionloss phenotype (Figure 8A). In addition, RT-PCR showed that the presence of pTRV2::ScSCPL2 was only detected in ScSCPL2silenced tissues (Figure 8B). Moreover, qRT-PCR results showed that the expression of ScSCPL2 in the tissues with functional loss was significantly lower than that in the negative control (Figure 8C). These results confirmed that TRV vectors could infiltrate cineraria successfully and that pTRV2::ScSCPL2 could knock down the expression of the endogenous ScSCPL2 gene to make leaves appear pink.

Then we used HPLC-MS/MS to identify the anthocyanin composition and content of ScSCPL2-silenced tissues. Four peaks existed in these tissues, named $\mathrm{B}^{\prime}, \mathrm{B}^{\prime}, \mathrm{B}^{\prime}$, and $\mathrm{B} 4^{\prime}$ (Figure 8D). Among them, the retention time, $\mathrm{UV}$-vis absorption maxima and protonated molecule results of $\mathrm{B}^{\prime}, \mathrm{B} 3^{\prime}$, and $\mathrm{B} 4^{\prime}$ were the same as those of B2, B3, B4 in VeB (Supplementary Table 6). The absorption peak around $330 \mathrm{~nm}$ of $\mathrm{B} 1$ ' was absent while it was existed in B1 (Supplementary Figure 2), which indicated absence of caffeoyl moiety in the molecule of $\mathrm{B1}^{\prime}$. In addition, we found that the $\mathrm{B}^{\prime}$ peak contained a [delphinidin] ${ }^{+}$fragment ion of $\mathrm{m} / \mathrm{z}$ 303 , and its molecular ion peak $[\mathrm{M}+\mathrm{H}]^{+}$was $\mathrm{m} / \mathrm{z} 875$, which was consistent with B1 (Dp3MalG-3'CafG, one glucosyl and one caffeoyl existed at the $3^{\prime}$ position of the $\mathrm{B}$ ring). Two intermediate fragment ions, $\mathrm{m} / \mathrm{z} 551\left([\mathrm{Dp} 3 \mathrm{MalG}+\mathrm{H}]^{+}\right)$and $\mathrm{m} / \mathrm{z} 713$ $\left(\left[\mathrm{Dp} 3 \mathrm{MalG}-3^{\prime} \mathrm{G}+\mathrm{H} \text { or Dp3MalG-7G }+\mathrm{H}\right]^{+}\right)$, were detected in
$\mathrm{B1}^{\prime}$, while only one fragment ion of $\mathrm{m} / \mathrm{z} 551\left([\mathrm{Dp} 3 \mathrm{MalG}+\mathrm{H}]^{+}\right)$ existed in $\mathrm{B} 1$, which indicated that a single glucosyl existed at the $3^{\prime}$ and 7-positions of $\mathrm{B1}^{\prime}$ simultaneously. Based on these UV-vis absorption spectra and protonated molecule data, we speculated the chemical structure of B1' was Dp3MalG-7G-3'G. After inhibiting the expression of ScSCPL2 in blue cineraria, the content of Dp3MalG-7CafG-3'CafG and Dp3MalG-7d(CafG)$3^{\prime} \mathrm{CafG}$ (cinerarin) decreased from 32.5to $3.01 \mu \mathrm{g} / \mathrm{g}$ and from 42.5 to $0.69 \mu \mathrm{g} / \mathrm{g}$, respectively, while Dp3MalG-7G-3' $\mathrm{G}$, which had no acylation at the $3^{\prime}$ or 7-position, accumulated at a high level $(43.2 \mu \mathrm{g} / \mathrm{g}$ ) (Figure 8E). These results showed that ScSCPL2 was a key structural gene mainly involved in the $3^{\prime}$ and 7-position acylation for blue flower formation in cineraria.

\section{Identification of MYBs Involved in the Regulation of Polyacylated Anthocyanin Biosynthesis}

The MYB-bHLH-WD40 complex plays an important role in regulating the transcription of structural genes involved in the anthocyanin biosynthesis pathway. The MYB transcription factor is extremely important in the MYB-bHLH-WD40 complex (Jaakola, 2013). According to the K-means analysis of differentially expressed genes, Two MYB transcription factors named ScMYB2 and ScMYB4 from cluster 8 were identified. Phylogenetic analysis indicated that ScMYB2 belonged to subfamily 4 of the R2R3-MYB transcription factor gene family, while ScMYB4 was clustered to the R3 MYB transcription factor gene family (Figure 9A). The expression profiles of these two MYB transcription factors were detected using qRT-PCR. The results showed that $S c M Y B 2$ and ScMYB4 were only highly expressed in $\mathrm{VeB}$, while they were barely detectable in $\mathrm{VeW}$ and VeC (Figure 9B). In addition, ScMYB2 and ScMYB4 were found to have expression patterns closely related to key structural genes, such as ScUGT1, ScUGT4, ScBGLU11, ScBGLU12, and ScSCPL2, which might be involved in the polyacylation modification of cinerarin, through gene coexpression network analysis, and these genes all belonged to cluster 8 of DEGs (Figure 9C). Based on the above results, ScMYB2 and ScMYB4 were selected as key candidate transcription factors in the regulation of the delphinidin biosynthesis pathway and polyacylation modification in blue cineraria.

\section{DISCUSSION}

\section{ScUGT1/4 and ScAGGT11/ScAGGT12 Might Be Involved in the Glycosylation of the $3^{\prime}$ - and 7-O-Position of Cinerarin}

Glycosyltransferases and acyltransferases involved in anthocyanin modification resulted in rich variation in flower colour. Glycosylation and acylation at the $3^{\prime}$ position of the B ring and 7-position of the $\mathrm{A}$ and $\mathrm{C}$ rings play very important roles in the formation of stable blue flower colour (Matsuba et al., 2010; Nishizaki et al., 2013). Cineraria is an important potted ornamental plant, and its blue flowers contain $3^{\prime}, 7$-polyacylated delphinidin-type anthocyanins, which has not been reported in 


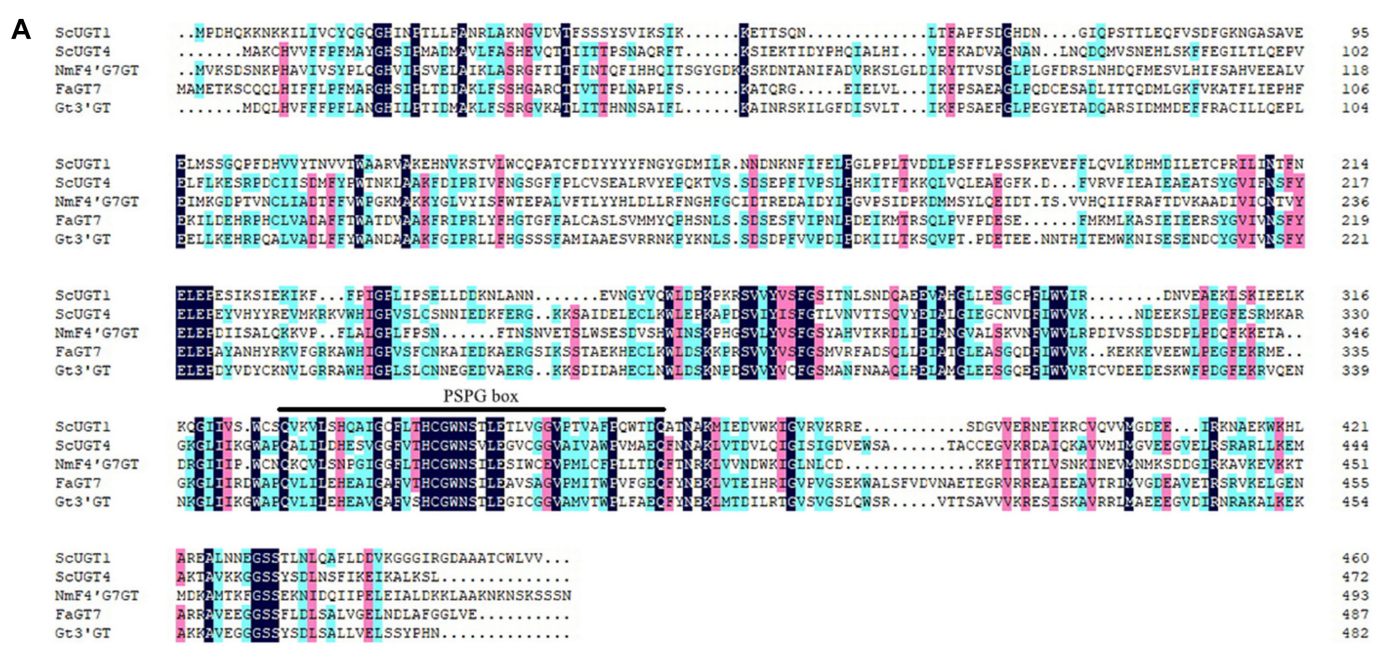

B

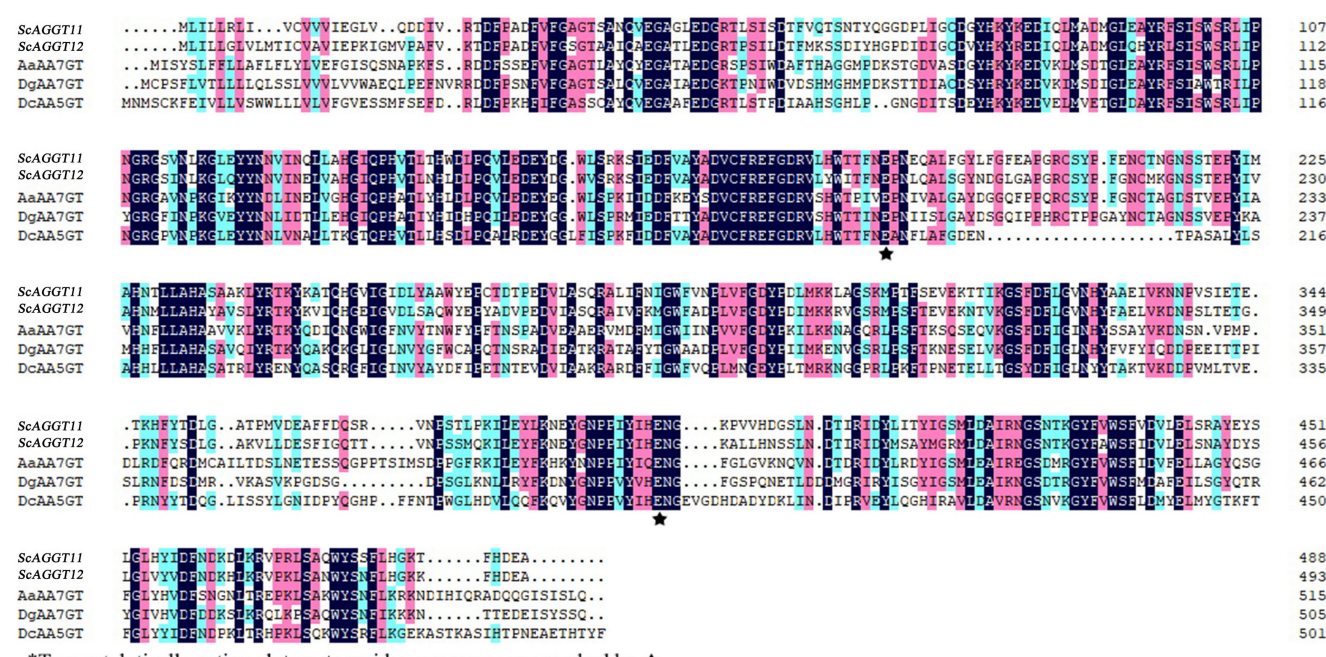

C

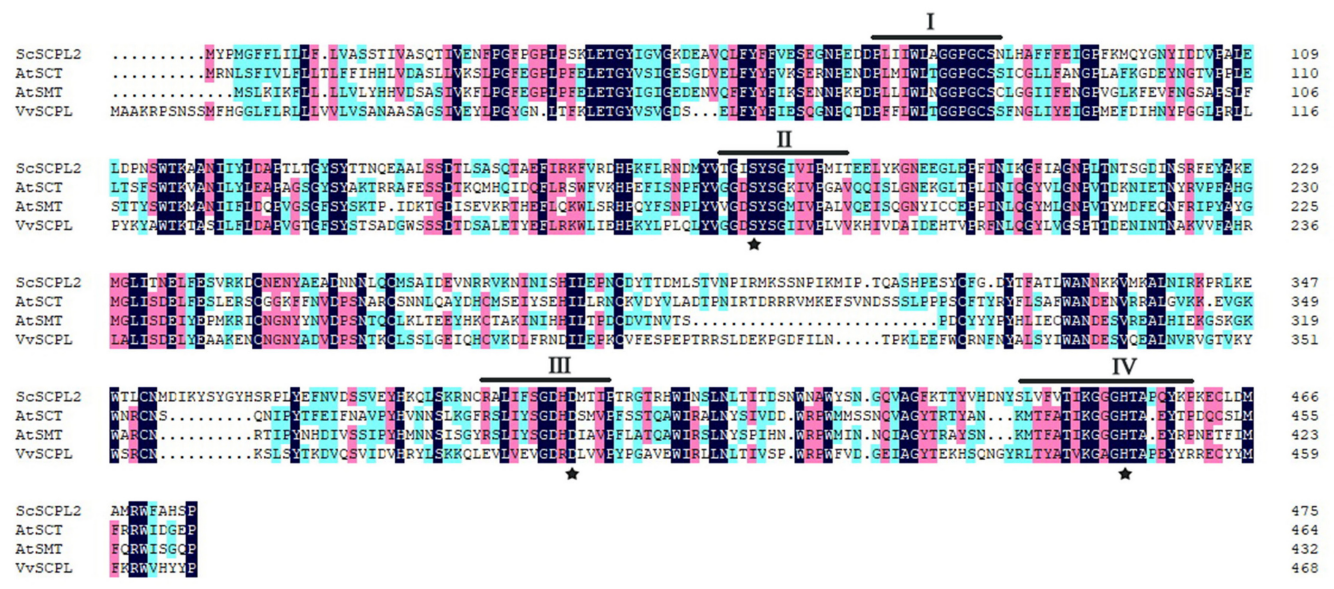

FIGURE 7 | Multiple alignments of amino acid sequences of ScUGT1, ScUGT4, ScBGLU11, ScBGLU12, and ScSCPL2. (A) Sequence alignment of ScUGT1 and ScUGT4 (Senecio cruentus), NmF4'G7GT (Nemophila menziesii), FaGT7 (Fragaria ananassa), and Gt3'GT (Gentiana triflora). (B) Sequence alignment of ScAGGT11 and ScAGGT12 (Senecio cruentus), AaAA7GT (Agapanthus africanus), DgAA7GT (Delphinium grandiflorum), and DcAA5GT (Dianthus caryophyllus). (C) Sequence alignment of SCSCPL2 (Senecio cruentus), AtSCT (Arabidopsis thaliana), AtSMT (Arabidopsis thaliana), and VvSCPL (Vitis vinifera). 
A

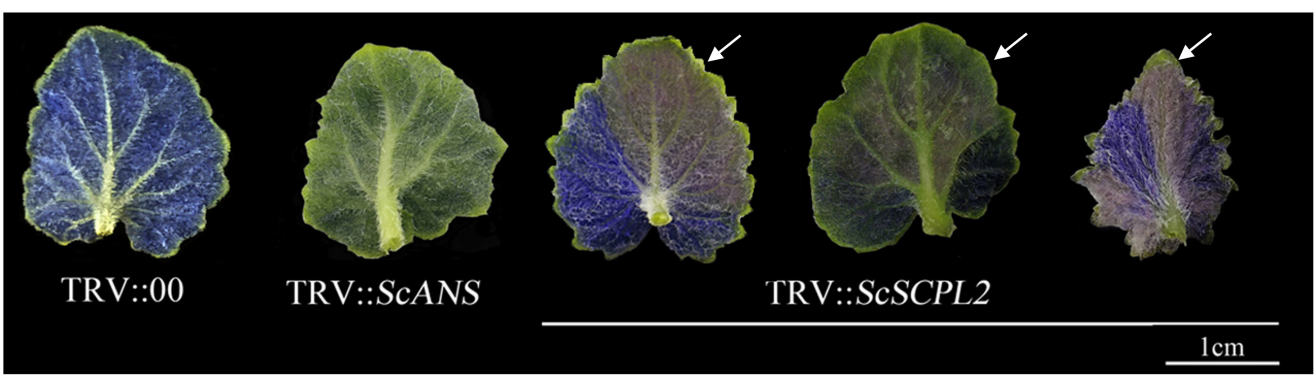

B
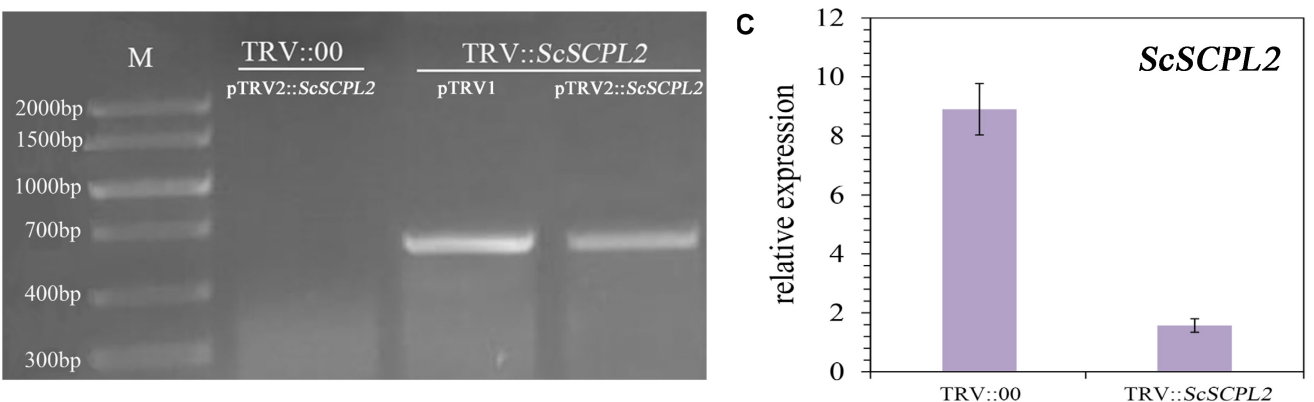

D

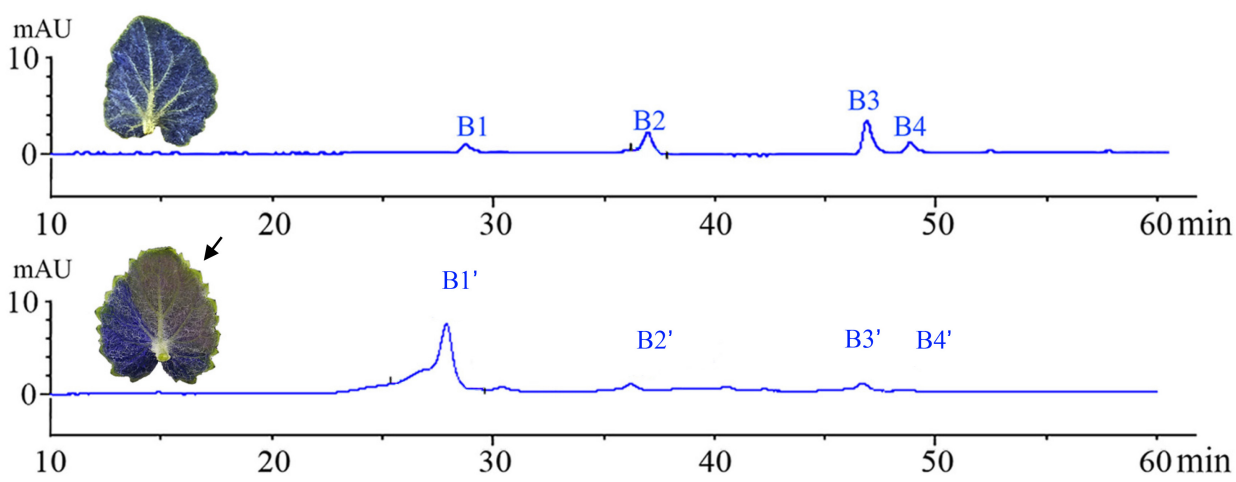

E

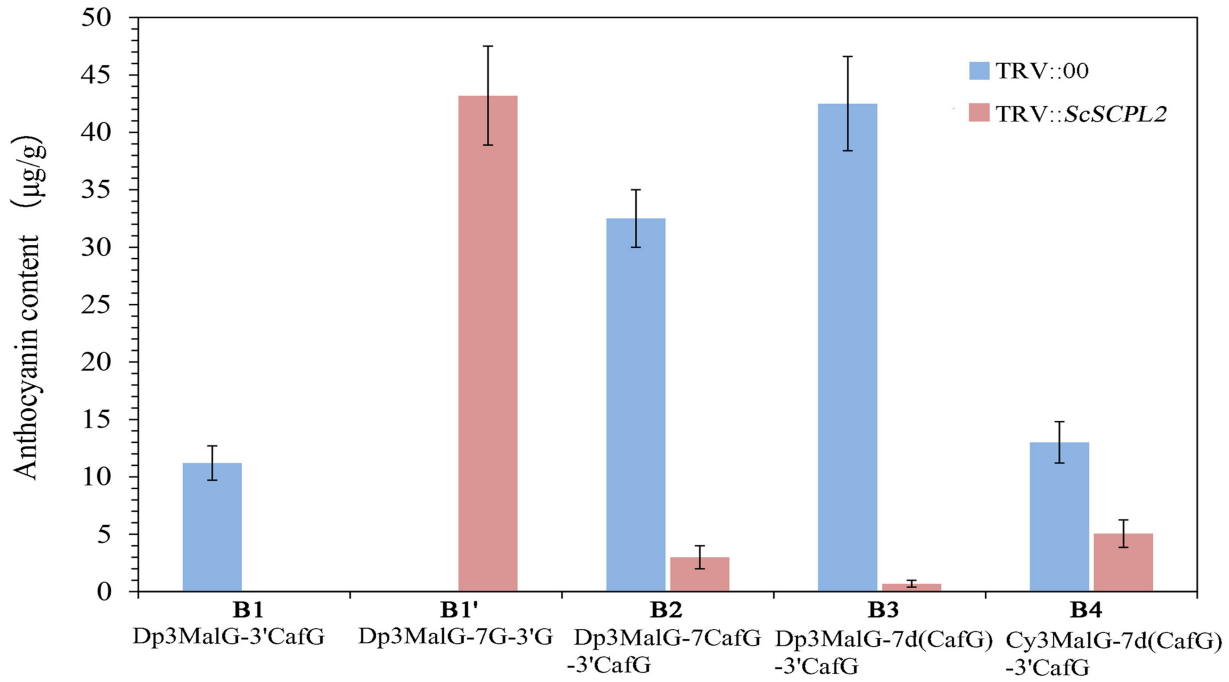

FIGURE 8 | Transient silencing of SCSCPL2 by VIGS in blue cineraria. (A) The phenotype of the leaves of blue cineraria whose ScSCPL2 gene was silenced by VIGS. (B) Detection of pTRV1 and pTRV2::SCSCPL2 in the silencing tissue of blue cineraria. (C) qRT-PCR analysis of ScSCPL2 both in the silencing tissue and negative control. (D) Anthocyanin composition in the leaves and SCSCPL2-silenced tissues of blue cineraria by HPLC analysis. (E) The content of anthocyanins in the leaves and SCSCPL2-silenced tissues of blue cineraria. 
A
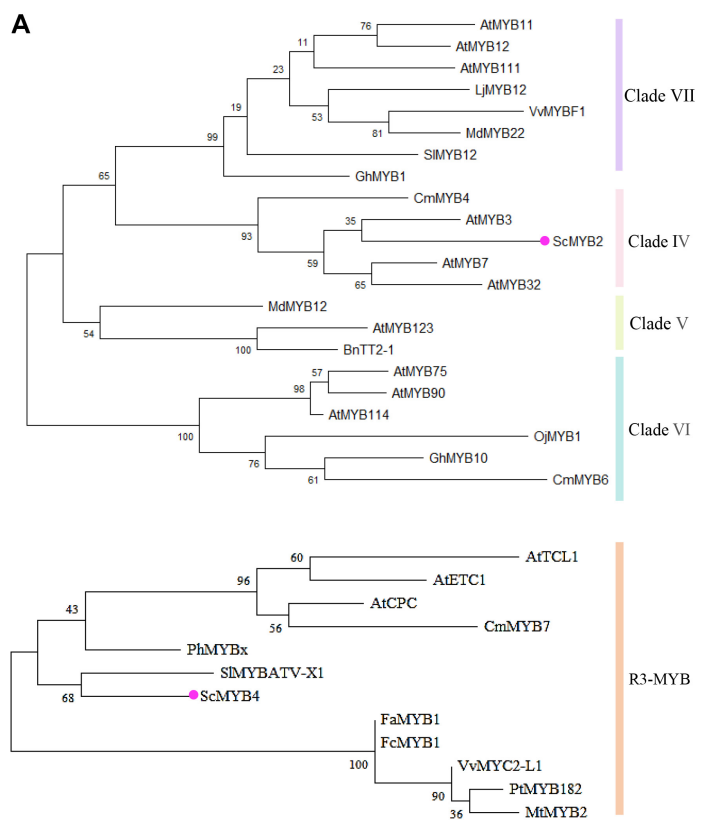

B
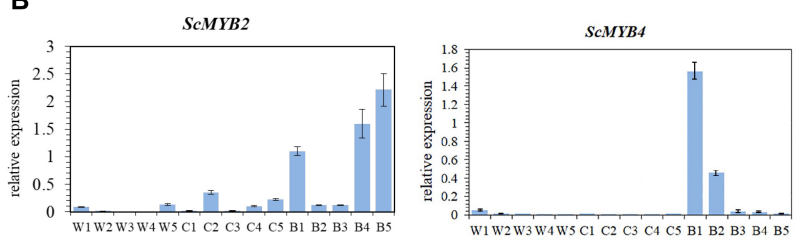

C
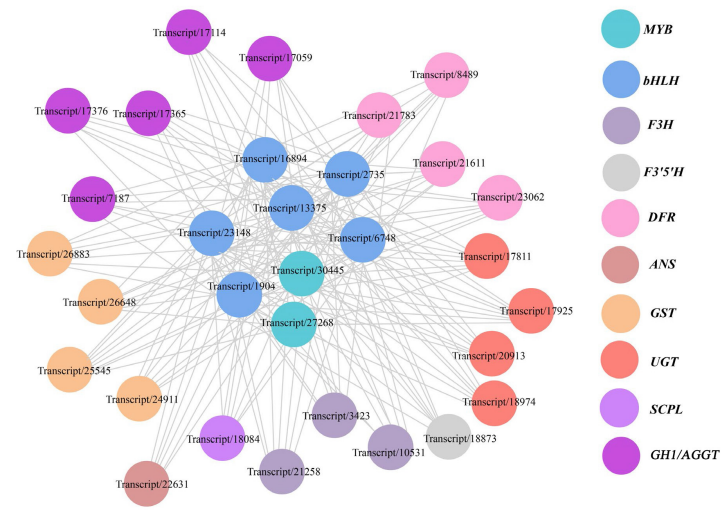

FIGURE 9 | Identification of MYBs involved in the regulation of polyacylated anthocyanin biosynthesis using bioinformatics and qRT-PCR analysis. (A) Amino acid sequences of SCMYB2 and SCMYB4 and arabidopsis MYBs belonging to the Clade IV-VII and R3-MYB and some MYB sequences from other plants were used to build a phylogenetic tree based on maximum likelihood, which was employed to infer the MYB candidates of cineraria involved in polyacylation modification. (B) The expression profiles of SCMYB2 and SCMYB4 during the ray florets developed were confirmed by qRT-PCR. The data were the mean \pm SD from three biological replicates. (C) The relationship between SCMYB2, SCMYB4 and the key structural genes related to delphinidin biosynthesis and polyacylation modification of cineraria in cluster 8 of DEGs was analyzed by gene co-expression network. The circles represented the transcripts, and the grey lines represented connectivity among the transcripts.

any other plants. The main anthocyanin in the blue colour of the ray florets was cinerarin, which contained one glucosyl and one caffeoyl at the $3^{\prime}$ position, two glucosyls and two caffeoyls at the 7-position (Goto et al., 1984; Suzuki et al., 2003), suggesting the important role of polyacylation in blue flower formation.

Glycosylation by GTs provides the basis of acylation by ATs. UGTs catalyse flavonoids to biosynthesize various glycoside derivatives, among which 3-/5-position modifications are the common skeleton of anthocyanins, while UGTs that are specific for $3^{\prime}$ or 7-position modifications can catalyse polyacylated anthocyanin biosynthesis (Sasaki and Nakayama, 2015). In this study, using comparative transcriptome analysis combined with gene expression, we found that ScUGT1 and ScUGT4 were highly expressed in the ray florets of $\mathrm{VeB}$ but almost not expressed in the ray florets of $\mathrm{VeW}$ and $\mathrm{VeC}$ (Figures 6D,E). Phylogenetic analysis has revealed that the positional specificities of the glycosylation modification catalysed by UGTs are closely related to the phylogenetic clades of the enzymes. We found that ScUGT1 was clustered with DgpHBAGT, which is involved in the synthesis of $p$-hydroxybenzoyl-glucose (pHBG), which acts as a zwitter donor in acylation and glucosylation in D. grandiflorum (Figure 6A) (Nishizaki et al., 2014). This result indicated that ScUGT1 might be involved in the synthesis of caffeoylglucose, a type of acyl group donor, in cineraria. Meanwhile, both ScUGT4 and GtUA3'GT belonged to Clade IIIa by phylogenetic analysis (Figure 6A). GtUA3'GT of G. triflora is involved in gentiodelphin (3G-5CafG-3' $3^{\prime}$ afG) biosynthesis, and the GtUA3'GT knockout lines accumulate delphinidin $3 \mathrm{G}-5 \mathrm{CafG}$ as the major flower pigment instead of gentiodelphin (Tasaki et al., 2019). Thus, we selected ScUGT4 as the key candidate gene that may catalyse the $3^{\prime}$ position glycosylation of cinerarin. However, the enzymatic function of ScUGT4 needs further verification.

Uridine diphosphate-dependent glycosyltransferase family 1 that specifically catalyse the 7-position glycosylation of anthocyanins have not yet been discovered, while UGTs that catalyse the 7-position glycosylation of other flavonoids have been characterized in blue Nemophila menziesii and Veronica persica. The 7-glycosylated flavonoids catalysed by NmF4'G7GT were used as anthocyanin copigments to produce a deeper and bluer colour through intramolecular stacking. The 7glycosylated flavonoids catalysed by VPUF7GAT complexed with metal ions and glycosylated anthocyanins also make plants appear blue (Ono et al., 2010; Okitsu et al., 2018). AGGT is another glycosyltransferase involved in the modification of anthocyanins. For example, the 7-polyacylated anthocyanin of delphinidin was synthesized via step-by-step enzymatic reactions of glycosylation catalysed by BGLUs (DgAA7GT and $D g A A 7 B G-G T)$ and $p$-hydroxybenzoylation catalysed by SCPL-AT (DgSCPL2) (Matsuba et al., 2010; Luang et al., 2013; Nishizaki et al., 2013). In this study, ScAGGT11 and ScAGGT12 were specifically highly expressed in the ray florets of $\mathrm{VeB}$ (Figures 6D,E). They are both clustered in the same clade as DgAA7GT (Figure 7B), which were speculated to be involved in the glycosylation of the 7-O-position of 
cinerarin in blue cineraria. However, the specific function needs further verification.

\section{ScSCPL2 Participated in the $3^{\prime}$ and 7-Position Acylation of Cinerarin}

There are two important gene families related to the acylation of anthocyanins: acyl-CoA-dependent acyltransferase BAHD and SCPL utilizing acyl-Glc as the donor molecule. The products of the BAHD family are mainly monoacylated anthocyanins (Bontpart et al., 2015). Suzuki et al. (2003) identified a BAHD-type AAT acyltransferase Sc3MaT in blue cineraria. The analysis of enzymatic properties showed that it specifically used malonyl-CoA as the donor and anthocyanin 3-O-glucoside as the acceptor to synthesize anthocyanin 3-O-glucose- $6^{\prime \prime}$-malonyl (Anthocyanin-3GMal). However, the $3^{\prime}$ and 7-position aromatic acyl modifications of anthocyanins are key for the formation of bright blue flowers of cineraria. At present, there are no studies on the mechanism of $3^{\prime}$ and 7-position acylation of cineraria.

In this study, we found that ScSCPL2, encoding an acylGlc-dependent acyltransferase in cineraria, showed specific high expression in $\mathrm{VeB}$ through comparative transcriptomic analysis combined with gene expression (Figures 6D,E). VIGS showed that the leaves of cineraria infiltrated with pTRV2::ScSCPL2 exhibited a function-loss phenotype, whose colour turned pink, at 30 days post-infiltration (Figure $\mathbf{8 A}$ ). In addition, the content of Dp3MalG-7CafG-3'CafG and Dp3MalG-7d(CafG)$3^{\prime} \mathrm{CafG}$ (cinerarin) decreased significantly after inhibiting the expression of ScSCPL2 in blue cineraria, while Dp3MalG-7G$3^{\prime} G$, which had no acylation at the $3^{\prime}$ or 7-position, accumulated at a high level (Figures 8D,E). Phylogenetic analysis revealed that SCSCPL2 belonged to the Clade Ia subfamily (Figure 6C), whose members mainly encoded acyltransferases to catalyse the acylation of flavonoids (including anthocyanins). For example, At2g23000, a member of the Clade Ia subfamily in A. thaliana, has been shown to use 1- $O$-sinapoyl- $\beta$-D-glucoses as substrates to transfer sinapoyl groups to cyanins to form sinapoylated cyanins (Fraser et al., 2007; Shi and Xie, 2014). CtA3'AT of C. ternatea could participate in $3^{\prime}$ position polyacylation by transferring the $p$-coumaroyl groups from $p$-coumaroylglucoses to anthocyanins (Noda et al., 2006). DgSCPL2 of D. grandiflorum, belonging to the Clade Ia subfamily, was involved in biosynthesizing 7-polyacylated anthocyanins combined with DgAA7GT and $D g A A 7 B G-G T$ (Nishizaki et al., 2013). Thus, we regarded ScSCPL2 as a key structural gene mainly involved in the $3^{\prime}$ and 7 -position acylation for blue flower formation in cineraria.

\section{ScMYB2 and ScMYB4 Might Be Involved in the Transcriptional Regulation of Polyacylation Modification}

MYB, bHLH, and WD40 are the major regulatory genes that regulate the expression of structural genes in the anthocyanin biosynthesis pathway. In recent years, many studies have found that MYB transcription factors also regulate the expression of genes specifically related to anthocyanin modification. In G. triflora, GtMYB3 could interact with GtbHLH1 to activate the expression of GtA5/3'AT (Nakatsuka et al., 2008). DcMYB113 was expressed specifically in the roots of purple carrot and was involved in regulating the biosynthesis of anthocyanins by activating the expression of DcUCGXT1 and DcSAT1, which encoded uridine diphosphate glycosyltransferase and SCPL acyltransferase (Xu et al., 2020). MYBA1 activated the expression of VvSCPL5, which was found in V. vinifera (Bontpart et al., 2018). This study found that ScMYB2 and ScMYB4 were highly expressed in $\mathrm{VeB}$, while almost no expression was detected in $\mathrm{VeW}$ and $\mathrm{VeC}$ by comparative transcriptomic and gene expression analysis (Figure 9B). According to K-means analysis and gene coexpression network analysis, ScMYB2, ScMYB4 and the key candidate structural genes ScUGT1, ScUGT4, ScAGGT11, ScAGGT12, and ScSCPL2, which might be involved in the polyacylation modification of cineraria, were found in cluster 8 (Figure 9C). Therefore, we speculated that ScMYB2 and ScMYB4 were involved in the transcriptional regulation of polyacylation modification in blue cineraria.

Phylogenetic analysis suggested that ScMYB2 was clustered into subfamily 4 in the R2R3-MYB transcription factor family (Figure 9A). Previous study found that members in subfamily 4 mainly regulate the biosynthesis of flavonoids in plants. In Petunia hybrida, PhMYB27 was known as a transcription factor regulating the biosynthesis of flavonoids and can inhibit the expression of PhAN1 and PhDFR with an EAR motif in its protein sequence (Albert et al., 2014). MYB165 and MYB194 interacted with $b H L H 131$ to inhibit the transcriptional activation effect of MYB134 on related genes, such as ANR, $D F R, L A R$, and ANS, in the flavonoid metabolism pathway in Populus tremula $\times$ tremuloides (Ma et al., 2018). Furthermore, members of subfamily 4 have also been found to be directly involved in the regulation of the phenylalanine metabolism pathway. AtMYB3 in A. thaliana combined with the $C 4 H$ promoter directly to inhibit its activation. In the AtMYB3overexpressing lines, the expression of CHS, 4CL1, and 4CL3 decreased significantly, which led to a reduction in the content of malic acid and anthocyanins (Zhou et al., 2017). The above studies showed that members in subfamily 4 of the R2R3-MYB transcription factor family have diverse functions in different plant species. Thus, we hypothesized that $S c M Y B 2$ had the ability to regulate the expression of ScUGT1, ScUGT4, ScAGGT11, ScAGGT12, and ScSCPL2. ScMYB4 belonged to the R3-MYB transcription factor family (Figure 9A), which usually functions as a key repressor in anthocyanin metabolism (Sara et al., 2018). In Chrysanthemum $\times$ morifolium, R3 MYB transcription CmMYB\# 7 inhibited the biosynthesis of anthocyanins by competing with $\mathrm{CmMYB6}$ to combine $\mathrm{CmbHLH} 2$ (Xiang et al., 2019). Therefore, we speculated that ScMYB4 may produce new transcriptional activation or inhibit the activity of other $M Y B$ inhibitors to induce cineraria accumulation of a large amount of cinerarin. However, the specific function of ScMYB4 needs further analysis.

\section{CONCLUSION}

In this study, we found that $3^{\prime}, 7$-polyacylated anthocyanin, cinerarin, was the main pigment component that determined the 
blue colour of ray florets of cineraria by HPLC-MS/MS analysis. Based on comparative transcriptomic analysis combined with RT- and qRT-PCR, we found two genes encoding uridine diphosphate glycosyltransferase, named ScUGT1 and ScUGT4; two genes encoding acyl-glucoside-dependent glucosyltransferases which belong to glycoside hydrolase family 1 (GH1), named ScAGGT11 and ScAGGT12; one gene encoding SCPL acyltransferase ScSCPL2; and two MYB transcriptional factor genes $S c M Y B 2$ and $S c M Y B 4$, that were specifically highly expressed in the ray florets of $\mathrm{VeB}$, which indicated that these genes may be involved in cinerarin biosynthesis. Among them, ScUGT1 was used to prepare the acyl donor for acylation by caffeic acid, ScUGT4 may be involved in the glycosylation of $3^{\prime}$ $O$-position. ScAGGT11 and ScAGGT12 were speculated to be involved in the glycosylation of 7-O-position. ScSCPL2 mainly participated in the $3^{\prime}$ and 7-position acylation of cinerarin. ScMYB2 and ScMYB4 may specifically regulate the expression of these modified genes. Besides, ScUGT23 and ScUGT39 that highly expressed in $\mathrm{VeC}$ and $\mathrm{VeB}$ may be involved in modifying the 3position glycosylation, which was the most conventional and common glycosylation of anthocyanins but not the key step for blue flower formation. These results will provide new insight into the molecular basis of the polyacylated anthocyanin biosynthesis mechanism in higher plants and are of great significance for blue flower molecular breeding of ornamental plants.

\section{REFERENCES}

Akagi, T., Katayama-Ikegami, A., and Yonemori, K. (2011). Proanthocyanidin biosynthesis of persimmon (Diospyros kaki Thunb.) fruit. Sci. Hortic. 130, 373-380. doi: 10.1016/j.scienta.2011.07.021

Albert, N. W., Davies, K. M., Lewis, D. H., Zhang, H., and Schwinn, K. E. (2014). A conserved network of transcriptional activators and repressors regulates anthocyanin pigmentation in eudicots. Plant Cell 26, 962-980. doi: 10.1105/tpc. 113.122069

Amoanimaa-Dede, H., Hongbo, Z., Kyereko, W. T., Yeboah, A., and Asiamah, C. A. (2019). Structure, functions and biosynthetic pathway of naturally occurring anthocyanin in sweet potato - a review. J. Plant Biochem. Physiol. 7:234. doi: 10.35248/2329-9029.19.7.234

Anders, S., and Huber, W. (2010). Differential expression analysis for sequence count data. Nat. Prec. 11:R106. doi: 10.1038/npre.2010.4282.1

Bontpart, T., Cheynier, V., Ageorges, A., and Terrier, N. (2015). BAHD or SCPL acyltransferase? What a dilemma for acylation in the world of plant phenolic compounds. New Phytol. 208, 695-707. doi: 10.1111/nph. 13498

Bontpart, T., Ferrero, M., Khater, F., Marlin, T., Vialet, S., Vallverdu-Queralt, A., et al. (2018). Focus on putative serine carboxypeptidase-like acyltransferases in grapevine. Plant Physiol. Biochem. 130, 356-366. doi: 10.1016/j.plaphy.2018. 07.023

Chen, J., Yang, H., Li, Z., Xu, N., Yu, B., Xu, J., et al. (2016). Construction of protein interaction network involved in lung adenocarcinomas using a novel algorithm. Oncol. Lett. 12, 1792-1800. doi: 10.3892/ol.2016. 4822

Chen, K., Du, L., Liu, H., and Liu, Y. (2019). A novel R2R3-MYB from grape hyacinth, MaMybA, which is different from MaAN2, confers intense and magenta anthocyanin pigmentation in tobacco. BMC Plant Biol. 19:390. doi: 10.1186/s12870-019-1999-0

Ciarkowska, A., Ostrowski, M., Starzyńska, E., and Jakubowska, A. (2018). Plant SCPL acyltransferases: multiplicity of enzymes with various functions in secondary metabolism. Phytochem. Rev. 18, 303-316. doi: 10.1007/s11101-0189593-x

\section{DATA AVAILABILITY STATEMENT}

The RNA-Seq data and Iso-Seq data have been deposited in NCBI under SRA accession codes: PRJNA674976.

\section{AUTHOR CONTRIBUTIONS}

$\mathrm{HH}$ and SD conceived and designed this study. YL, YC, HH, and JR performed the experiments. CL, YL, HH, and JQ carried out the data analysis. CL, HH, YL, and FQ wrote this manuscript. All authors read and approved the final manuscript.

\section{FUNDING}

This work was supported by the National Natural Science Foundation of China (grant no. 32071826).

\section{SUPPLEMENTARY MATERIAL}

The Supplementary Material for this article can be found online at: https://www.frontiersin.org/articles/10.3389/fpls.2021. 640746/full\#supplementary-material

Conesa, A., Götz, S., García-Gómez, J. M., Terol, J., Talón, M., and Robles, M. (2005). Blast2GO: auniversal tool for annotation, visualization and analysis in functional genomics research. Bioinformatics 21, 3674-3676. doi: 10.1093/ bioinformatics/bti610

Doncheva, N. T., Morris, J. H., Gorodkin, J., and Jensen, L. J. (2018). Cytoscape stringapp: network analysis and visualization of proteomics data. J. Proteome Res. 18, 623-632. doi: 10.1101/438192

Elmore, M. G., Banerjee, S., Pedley, K. F., Ruck, A., and Whitham, S. A. (2020). De novo transcriptome of Phakopsora pachyrhizi uncovers putative effector repertoire during infection. Physiol. Mol. Plant Pathol. 110:101464. doi: 10. 1016/j.pmpp.2020.101464

Felsenstein, J. (1985). Confidence limits on phylogenies: an approach using the bootstrap. Evolution 39, 783-791. doi: 10.1111/j.1558-5646.1985.tb0 0420.x

Fraser, C. M., Rider, L. W., and Chapple, C. (2005). An expression and bioinformatics analysis of the Arabidopsis serine carboxypeptidase-like gene family. Plant Physiol. 138, 1136-1148. doi: 10.1104/pp.104.057950

Fraser, C. M., Thompson, M. G., Shirley, A. M., Ralph, J., Schoenherr, J. A., Sinlapadech, T., et al. (2007). Related arabidopsis serine carboxypeptidaselike sinapoylglucose acyltransferases display distinct but overlapping substrate specificities. Plant Physiol. 144, 1986-1999. doi: 10.1104/pp.107. 098970

Goto, T., Kondo, T., Kawai, T., and Tamura, H. (1984). Structure of cinerarin, a tetra-acylated anthocyanin isolated from the blue garden cineraria, Senecio cruentus. Tetrahedron Lett. 25, 6021-6024. doi: 10.1016/S0040-4039(01) 81749-4

Hui, Z., Qian, P., Zhao, J., Albert, O., Fei, R., Liao, L., et al. (2016). Multiple R2R3-MYB transcription factors involved in the regulation of anthocyanin accumulation in Peach flower. Front. Plant Sci. 7:1557. doi: 10.3389/fpls.2016. 01557

Jaakola, L. (2013). New insights into the regulation of anthocyanin biosynthesis in fruits. Trends Plant Sci. 18, 477-483. doi: 10.1016/j.tplants.2013.06.003

Jia, Y., Selva, C., Zhang, Y., Li, B., McFawn, L. A., Broughton, S., et al. (2020). Uncovering the evolutionary origin of blue anthocyanins in cereal grains. Plant J. 101, 1057-1074. doi: 10.1111/tpj.14557 
Jin, X. H., Huang, H., Wang, L., Sun, Y., and Dai, S. L. (2016). Transcriptomics and metabolite analysis reveals the molecular mechanism of anthocyanin biosynthesis branch pathway in different Senecio cruentus cultivars. Front. Plant Sci. 7:1307. doi: 10.3389/fpls.2016.01307

Li, B., and Dewey, C. N. (2011). RSEM: accurate transcript quantification from RNA-seq data with or without a reference genome. BMC Bioinform. 12:323. doi: 10.1186/1471-2105-12-323

Li, B. J., Zheng, B. Q., Wang, J. Y., Tsai, W. C., Lu, H. C., Zou, L. H., et al. (2020). New insight into the molecular mechanism of colour differentiation among floral segments in orchids. Commun. Biol. 3:89.

Li, J., Lease, K. A., Tax, F. E., and Walker, J. C. (2001). BRS1, a serine carboxypeptidase, regulates BRI1 signaling in Arabidopsis thaliana. Proc. Natl. Acad. Sci. U.S.A. 98, 5916-5921. doi: 10.1073/pnas.091065998

Li, Y. J., Liu, Y. T., Qi, F. T., Deng, C. Y., Lu, C. F., Huang, H., et al. (2019). Establishment of virus-induced gene silencing system and functional analysis of ScbHLH17 in Senecio cruentus. Plant Physiol. Biochem. 147, 272-279. doi: 10.1016/j.plaphy.2019.12.024

Lin, Z. Y., Damaris, R. N., Shi, T., Li, J. J., and Yang, P. F. (2018). Transcriptomic analysis identifies the key genes involved in stamen petaloid in lotus (Nelumbo nucifera). BMC Genomics 19:554. doi: 10.1186/s12864-0184950-0

Liu, Y., Tikunov, Y., Schouten, R. E., Marcelis, L. F., Visser, R., et al. (2018), Anthocyanin biosynthesis and degradation mechanisms in solanaceous vegetables: a review. Front. Chem. 6:52. doi: 10.3389/fchem.2018. 00052

Livak, K. J., and Schmittgen, T. D. (2001). Analysis of relative gene expression data using real-time quantitative PCR and the 2(-Delta Delta $\mathrm{C}(\mathrm{T})$ ) Method. Methods 25, 402-408. doi: 10.1006/meth.2001.1262

Lu, C. F., Pu, Y., Liu, Y. T., Li, Y. J., Qu, J. P., Huang, H., et al. (2019). Comparative transcriptomics and weighted gene co-expression correlation network analysis (WGCNA) reveal potential regulation mechanism of carotenoid accumulation in Chrysanthemum $\times$ morifolium. Plant Physiol. Biochem. 142, 415-428. doi: 10.1016/j.plaphy.2019.07.023

Luang, S., Cho, J. I., Mahong, B., Opassiri, R., Akiyama, T., Phasai, K., et al. (2013). Rice Os9BGlu31 is a transglucosidase with the capacity to equilibrate phenylpropanoid, flavonoid, and phytohormone glycoconjugates. J. Biol. Chem. 288, 10111-10123. doi: 10.1074/jbc.M112.423533

Ma, D. W., Reichelt, M., Yoshida, K., Gershenzon, J., and Constabel, C. P. (2018). Two R2R3-MYB proteins are broad repressors of flavonoid and phenylpropanoid metabolism in poplar. Plant J. 96, 949-965. doi: 10.1111/tpj. 14081

Matsuba, Y., Nobuhiro, S., Masayuki, T., Masachika, O., Yutaka, A., Emi, O., et al. (2010). A novel glucosylation reaction on anthocyanins catalyzed by acyl-glucose-dependent glucosyltransferase in the petals of carnation and delphinium. Plant Cell 22, 3374-3389. doi: 10.1105/tpc.110.077487

Milkowski, C., and Strack, D. (2010). Sinapate esters in brassicaceous plants: biochemistry, molecular biology, evolution and metabolic engineering. Planta 232, 19-35. doi: 10.1007/s00425-010-1168-Z

Moriya, Y., Itoh, M., Okuda, S., Yoshizawa, A. C., and Kanehisa, M. (2007). KAAS: an automatic genome annotation and pathway reconstruction server. Nucleic Acids Res. 35, W182-W185. doi: 10.1093/nar/gkm321

Moura, D. S., Bergey, D. R., and Ryan, C. A. (2001). Characterization and localization of a wound-inducible type I serine-carboxypeptidase from leaves of tomato plants (Lycopersicon esculentum Mill.). Planta 212, 222-230. doi: $10.2307 / 23386107$

Nakajima, J. I., Tanaka, Y., Yamazaki, M., and Saito, K. (2001). Reaction mechanism from leucoanthocyanidin to anthocyanidin 3-glucoside, a key reaction for coloring in anthocyanin biosynthesis. J. Biol. Chem. 276, 25797-25803. doi: 10.1074/jbc.M100744200

Nakatsuka, T., Haruta, K. S., Pitaksutheepong, C., Abe, Y., and Nishihara, M. (2008). Identification and characterization of R2R3-MYB and bHLH transcription factors regulating anthocyanin biosynthesis in Gentian flowers. Plant Cell Physiol. 49, 1818-1829. doi: 10.1093/pcp/pcn163

Nishizaki, Y., Sasaki, N., Yasunaga, M., Miyahara, T., Okamoto, E., Okamoto, M., et al. (2014). Identification of the glucosyltransferase gene that supplies the p-hydroxybenzoyl-glucose for 7-polyacylation of anthocyanin in delphinium. J. Exp. Bot. 65, 2495-2506. doi: 10.1093/jxb/eru134
Nishizaki, Y., Yasunaga, M., Okamoto, E., Okamoto, M., Hirose, Y., Yamaguchi, M., et al. (2013). p-hydroxybenzoyl-glucose is a zwitter donor for the biosynthesis of 7-polyacylated anthocyanin in delphinium. Plant Cell 25, 4150-4165. doi: $10.1105 /$ tpc. 113.113167

Noda, N. (2018). Recent advances in the research and development of blue flowers. Breed. Sci. 68, 79-87. doi: 10.1270/jsbbs. 17132

Noda, N., Kazuma, K., Sasaki, T., Kogawa, K., and Suzuki, M. (2006). Molecular cloning of 1-O-acylglucose dependent anthocyanin aromatic acyltransferase in ternatin biosynthesis of butterfly pea (Clitoria ternalea). Plant Cell Physiol. 47, s109. doi: $10.14841 /$ jspp.2006.0.341.0

Okitsu, N., Matsui, K., Horikawa, M., Sugahara, K., and Tanaka, Y. (2018). Identification and characterization of novel Nemophila menziesii flavone glucosyltransferases that catalyze biosynthesis of flavone 7,4'-O-diglucoside, a key component of blue metalloanthocyanins. Plant Cell Physiol. 59, 2075-2085. doi: $10.1093 / \mathrm{pcp} / \mathrm{pcy} 129$

Ono, E., Ruike, M., Iwashita, T., Nomoto, K., and Fukui, Y. (2010). Copigmentation and flavonoid glycosyltransferases in blue Veronica persica flowers. Phytochemistry 71, 726-735. doi: 10.1016/j.phytochem.2010.02.008

Pu, Y., Huang, H., Wen, X. H., Lu, C. F., Zhang, B. H., Gu, X. Q., et al. (2020). Comprehensive transcriptomic analysis provides new insights into the mechanism of ray floret morphogenesis in chrysanthemum. BMC Genomics 21:728. doi: 10.1186/s12864-020-07110-y

Sara, C., Pierdomenico, P., and Silvia, G. (2018). The atroviolacea gene encodes an R3-MYB protein repressing anthocyanin synthesis in tomato plants. Front. Plant Sci. 9:830. doi: 10.3389/fpls.2018.00830

Sasaki, N., and Nakayama, T. (2015). Achievements and perspectives in biochemistry concerning anthocyanin modification for blue flower coloration. Plant Cell Physiol. 56, 28-40. doi: 10.1093/pcp/pcu097

Shi, M. Z., and Xie, D. Y. (2014). Biosynthesis and metabolic engineering of anthocyanins in Arabidopsis thaliana. Recent Patents Biotechnol. 8, 47-60. doi: 10.2174/1872208307666131218123538

Sun, W., Li, C. H., Wang, L. S., and Dai, S. L. (2010). Accumulation and variation of anthocyanins in blue flowers of Senecio cruentus at different flowering stages. J. Beijing Forest. Univ. 32, 128-134. doi: 10.1080/00949651003724790

Suzuki, H., Sawada, S. Y., Yonekura-Sakakibara, K., Nakayama, T., Yamaguchi, M. A., and Nishino, T. (2003). Identification of a cDNA encoding malonylCoenzyme A: anthocyanidin 3-O-glucoside 6"-O-malonyltransferase from Cineraria (Senecio cruentus) flowers. Plant Biotechnol. 20, 229-234. doi: 10. 5511/plantbiotechnology.20.229

Tanaka, Y., and Brugliera, F. (2013). Review article: flower colour and cytochromes P450. Philos. Trans. R. Soc. Lond. B Biol. Sci. 368, 20120432. doi: 10.1098/rstb. 2012.0432

Tasaki, K., Higuchi, A., Watanabe, A., Sasaki, N., and Nishihara, M. (2019). Effects of knocking out three anthocyanin modification genes on the blue pigmentation of gentian flowers. Sci. Rep. 9:15831. doi: 10.1038/s41598-01951808-3

Xiang, L. L., Liu, X. F., Li, H., Yin, X. R., Grierson, D., Li, F., et al. (2019). CmMYB\#7, an R3 MYB transcription factor, acts as a negative regulator of anthocyanin biosynthesis in chrysanthemum. J. Exp. Bot. 70, 3111-3123. doi: $10.1093 / \mathrm{jxb} / \mathrm{erz} 121$

Xu, Z. S., Yang, Q. Q., Feng, K., Yu, X., and Xiong, A. S. (2020). DcMYB113, a rootspecific R2R3-MYB, conditions anthocyanin biosynthesis and modification in carrot. Plant Biotechnol. J. 18, 1585-1597. doi: 10.1111/pbi.13325

Yang, J., Yu, H., Liu, B. H., Zhao, Z. M., Liu, L., Ma, L. X., et al. (2013). DCGL v2.0: an R package for unveiling differential regulation from differential Co-expression. PLoS One 8:e79729. doi: 10.1371/journal.pone.0079729

Yonekura-Sakakibara, K., and Hanada, K. (2011). An evolutionary view of functional diversity in family 1 glycosyltransferases. Plant J. 66, 182-193. doi: 10.1111/j.1365-313X.2011.04493.x

Yoshida, K., Mori, M., and Kondo, T. (2009). Blue flower color development by anthocyanins: from chemical structure to cell physiology. Nat. Prod. Rep. 26, 884-915. doi: 10.1039/b800165k

Yoshida, K., and Negishi, T. (2013). The identification of a vacuolar iron transporter involved in the blue coloration of cornflower petals. Phytochemistry 94, 60-67. doi: 10.1016/j.phytochem.2013.04.017

Zhao, D. Q., Luan, Y. T., Xia, X., Shi, W. B., Tang, Y. H., and Tao, J. (2020). Lignin provides mechanical support to herbaceous peony (Paeonia 
lactiflora Pall.) stems. Hortic. Res. 7:213. doi: 10.1038/s41438-02000451-5

Zhou, M. L., Zhang, K. X., Sun, Z. M., Yan, M. L., and Wu, Y. M. (2017). LNK1 and LNK2 corepressors interact with the MYB3 transcription factor in phenylpropanoid biosynthesis. Plant Physiol. 174, 1348-1358. doi: 10.1104/pp. 17.00160

Ziegler-Graff, V., Guilford, P. J., and Baulcombe, D. C. (1991). Tobacco rattle virus RNA-1 29K gene product potentiates viral movement and also affects symptom induction in tobacco. Virology 182, 145-155. doi: 10.1016/0042-6822(91) 90658-X
Conflict of Interest: The authors declare that the research was conducted in the absence of any commercial or financial relationships that could be construed as a potential conflict of interest.

Copyright $\odot 2021 \mathrm{Lu}, \mathrm{Li}, \mathrm{Cui}$, Ren, Qi, Qu, Huang and Dai. This is an open-access article distributed under the terms of the Creative Commons Attribution License (CC BY). The use, distribution or reproduction in other forums is permitted, provided the original author(s) and the copyright owner(s) are credited and that the original publication in this journal is cited, in accordance with accepted academic practice. No use, distribution or reproduction is permitted which does not comply with these terms. 